\title{
Charge-transfer induced EUV and soft X-ray emissions in the heliosphere ${ }^{\star}$
}

\author{
D. Koutroumpa ${ }^{1}$, R. Lallement ${ }^{1}$, V. Kharchenko ${ }^{2}$, A. Dalgarno ${ }^{2}$, R. Pepino ${ }^{2}$, V. Izmodenov ${ }^{3}$, and E. Quémerais ${ }^{1}$ \\ 1 Service d'Aéronomie du CNRS, 91371 Verrières-le-Buisson, France \\ e-mail: dimitra.koutroumpa@aerov.jussieu.fr \\ 2 Harvard-Smithsonian Center for Astrophysics, 60 Garden Street, Cambridge, MA 02138, USA \\ ${ }^{3}$ Lomonosov Moscow State University, Department of Aeromechanics and Gas Dynamics, Faculty of Mechanics and Mathematics, \\ Moscow 119899, Russia
}

Received 22 Mars 2006 / Accepted 28 July 2006

\begin{abstract}
Aims. We study the EUV/soft X-ray emission generated by charge transfer between solar wind heavy ions and interstellar neutral atoms and variations of the X-ray intensities and spectra with the line of sight direction, the observer location, the solar cycle phase and the solar wind anisotropies, and a temporary enhancement of the solar wind similar to the event observed by Snowden et al. (2004) during the XMM-Hubble Deep Field North exposure.

Methods. Using recent observations of the neutral atoms combined with updated cross-sections and cascading photon spectra we have computed self-consistent distributions of interstellar hydrogen, helium and highly charged solar wind ions for a stationary solar wind and we have constructed monochromatic emission maps and spectra. We have evaluated separately the contribution of the heliosheath and heliotail, and included X-ray emission of the excited solar wind ions produced in sequential collisions to the signal.

Results. In most practicable observations, the low and medium latitude X-ray emission is significantly higher at minimum activity than at maximum, especially around December. This occurs due to a strong depletion of neutrals during the high activity phase, which is not compensated by an increase of the solar wind flux. For high latitudes the emission depends on the ion species in a complex way. Intensity maps are in general significantly different for observations separated by six-month intervals. Secondary ions are found to make a negligible contribution to the X-ray line of sight intensities, because their density becomes significant only at large distances. The contribution of the heliosheath-heliotail is always smaller than $5 \%$.

We can reproduce both the intensity range and the temporal variation of the XMM-HDFN emission lines in the $0.52-0.75 \mathrm{keV}$ interval, using a simple enhanced solar wind spiral stream. This suggests a dominant heliospheric origin for these lines, before, during and also after the event.
\end{abstract}

Key words. solar wind - X-rays: diffuse background - ISM: general - galaxy: halo - X-rays: ISM - ISM: supernova remmants

\section{Introduction}

That objects as cold as comets emit copious X-rays was a surprising discovery (Lisse et al. 1996). The emission mechanism, first proposed by Cravens (1997), is now convincingly demonstrated as a major source of cometary and planetary X-rays. Charge exchange $(\mathrm{CX})$ collisions of highly charged ions of the solar wind (SW) with the neutral atoms and molecules from the coma produce cascades of photons in the extreme ultraviolet (EUV) and soft X-ray regions (Häberli et al. 1997; Krasnopolsky 1997; Kharchenko \& Dalgarno 2000; Schwadron \& Cravens 2002).

Cox (1998) pointed out that neutral interstellar (hereafter IS) and geocoronal atoms would also undergo $\mathrm{CX}$ with the $\mathrm{SW}$ ions, and generate soft X-rays throughout the heliosphere. The heliospheric emission has been subsequently modelled by Cravens et al. (2001) and Robertson et al. (2001), who explained satisfactorily the global correlation observed by Freyberg (1994, 1998) between the so-called Long Term Enhancements (LTE's) measured by the ROSAT satellite and strong solar wind events. The geocoronal emission has been investigated by

* Figures 9-13 are only available in electronic form at http://www . aanda. org
Robertson \& Cravens (2003a,b) and measured during Chandra observations of the dark moon (Wargelin et al. 2004). Earlier, Gruntman (1998) modelled the EUV emission produced by the recombination of alpha particles of the SW colliding with interstellar atoms, and showed the substantial influence of the solar wind characteristics on the emission.

The intensity of the soft X-rays in the heliosphere was first estimated by Cravens (2000), who found it to be of the same order as the soft X-ray emission from the so-called Local Bubble (hereafter LB), the 100 parsecs wide cavity surrounding the Sun, filled with tenuous hot $\left(10^{6} \mathrm{~K}\right)$ gas (Snowden et al. 1994, 1999). More generally, for faint and diffuse astronomical X-ray sources the CX heliospheric emission may contaminate significantly the $\mathrm{X}$-ray spectra below $1.5 \mathrm{keV}$ as has been demonstrated by a long duration XMM-Newton exposure towards the Hubble Deep Field-North (Snowden et al. 2004, hereafter SCK04).

The cosmic diffuse $\mathrm{X}$ - ray emission is the sum of extragalactic, halo and LB contributions (Kuntz \& Snowden 2000). It is difficult to separate out the CX emissions from those of the Local Bubble (LB). Shadowing is of limited use because of the absence of clouds of sufficient density and emission lines dominate the continuum in the CX spectrum and the LB spectrum. 
As seen from the Sun, heliospheric CX emission maxima will be aligned along the interstellar wind axis (Cox 1998). The ROSAT 6-month survey necessary to reconstruct full sky soft $\mathrm{X}$-ray maps did not show this alignment so that low upper limits to the local emission were derived. However, it has been demonstrated that parallax effects connected with the ROSAT all-sky survey geometry destroy the axial symmetry and cause a much smoother emission pattern, so that substantial contamination by CX is not excluded (Lallement 2004).

Robertson \& Cravens (2003a,b) constructed sky maps of the heliospheric and geocoronal CX intensity, using global efficiency factors representing all ions at once. Pepino et al. (2004) carried out similar calculations of the spectra emitted by the individual ions and of the corresponding power densities taking separate account of collisions of hydrogen and helium and distinguishing between the fast and slow solar winds.

We extend the study to the calculation of line of sight (LOS) spectral emission maps. We explore potential sources of variability of the received signals and quantify the resulting intensities. We take into account the observer location, the solar cycle phase, the LOS and we calculate the intensities of the strongest emission lines and the contributions of the outer heliosphere. We investigate the contribution of secondary ions created sequentially by $\mathrm{CX}$. We examine the effects of temporary solar wind enhancements and solar rotation (Cravens 2000) using a realistic model of the expanding solar wind. Observations of the soft diffuse X-ray background with the XMM-Newton telescope are analysed.

In Sect. 2 we describe the model developed for the calculation of the X-ray emission. In Sect. 3, we present the heliospheric $\mathrm{X}$-ray emission maps derived from the model and discuss the effects of the solar cycle, observing location on the emission pattern. In Sect. 3.3 we evaluate the contribution of the secondary ions and in Sect. 3.4 the contributions of the heliosheath and heliotail. In Sect. 4, we present X-ray and EUV spectra for lines of sight at different helio-ecliptic latitudes, corresponding to slow and fast solar winds, and for neutral gas distributions that depend on solar activity conditions. In Sect. 5 we simulate the temporal variations of the heliospheric X-ray emission generated by a solar impulsive event for solar wind conditions and a geometry corresponding to the XMM-Newton Hubble Deep Field-North (HDFN) exposure of June 1, 2001. The model results are compared with the XMM-Newton observations of the diffuse background spectra by SCK04. Finally, in the last Sect. 6, we summarize and discuss the results.

\section{Description of the model}

Our simulation of the heliospheric CX emission has four steps. The first is the computation of the density distribution of IS $\mathrm{H}$ and $\mathrm{He}$ atoms in response to the solar wind and solar EUV conditions for solar minimum and maximum activity. In the second step, these density distributions are used to calculate densities of heavy solar wind ions $\left(\mathrm{X}^{Q+}\right)$, modulated by collisions with the neutral heliospheric gas. In the third step we use the selfconsistent density grids of $\mathrm{H}$ and $\mathrm{He}$ neutral atoms and solar wind ions to calculate the X-ray emissivity due to the CX collisions. Finally these emissivities are used to calculate the total intensity along all lines of sight.

\subsection{Hydrogen and helium density grids}

In order to calculate the neutral $\mathrm{H}$ density distribution we have adapted a so-called classical "hot model" used in computing the interplanetary-interstellar H Lyman- $\alpha$ emission (Lallement et al. 1985a,b). The IS hydrogen flow, after crossing the heliospheric interface, is described as a single Maxwellian flow. The density distribution reflects the action of gravity, radiation pressure and losses due to solar wind CX and solar EUV ionization and recognises the latitudinal anisotropy of the loss terms. Although the $\mathrm{CX}$ with $\mathrm{H}^{+}$is not an $\mathrm{H}$ loss process, the newly created $\mathrm{H}$ atoms are expanding radially at a high velocity and their contribution to the density is negligible. Thus, we treat resonant $\mathrm{CX}$ as if it were a pure ionization process of the IS hydrogen gas. The parameters specifying the IS neutral hydrogen are: $n_{\mathrm{H}}($ at $100 \mathrm{AU})=$ $0.1 \mathrm{~cm}^{-3}, T=13000 \mathrm{~K}, V_{\mathrm{o}}=21 \mathrm{~km} \mathrm{~s}^{-1}, \lambda_{\mathrm{UW}}=252.3^{\circ}$, $\beta_{\mathrm{UW}}=8.5^{\circ}$ (Lallement et al. 2005), where $\lambda_{\mathrm{UW}}$ and $\beta_{\mathrm{UW}}$ are the helio-ecliptic longitude $(\lambda)$ and latitude $(\beta)$ respectively for the upwind direction of the incoming neutral $\mathrm{H}$ flow. We employ a density grid $n_{\mathrm{H}}[r, \lambda, \beta]$ with a $1^{\circ} \times 1^{\circ}$ resolution for $\lambda, \beta$ and for $r$ a variable step increasing with distance $r$ from the Sun, from $\delta r=0.3 \mathrm{AU}$ at the earth orbit up to $\delta r=9 \mathrm{AU}$ at $100 \mathrm{AU}$.

Helium density distributions in the inner heliosphere have been computed with the model developed by Lallement et al. (2004). The helium distributions are described by the classical kinetic model, with the parameters: $n_{\mathrm{He}}($ at $100 \mathrm{AU})=$ $0.015 \mathrm{~cm}^{-3}, T=6300 \mathrm{~K}, V_{\mathrm{o}}=26.2 \mathrm{~km} \mathrm{~s}^{-1}, \lambda_{\mathrm{DW}}=74.7^{\circ}$, $\beta_{\text {DW }}=-5.3^{\circ}$ (Witte 2004; Vallerga et al. 2004; Gloeckler et al. 2004). Resolution in $\theta$ is $1^{\circ}$. Distance steps are varying logarithmically in the sunward direction and they are very small near the Sun, where the helium is still dense and the emissivity due to $\mathrm{He}$ is high and varying rapidly.

Solar activity influences differently the heliospheric distributions of $\mathrm{H}$ and $\mathrm{He}$ atoms. The ratio, $\mu$, of radiation pressure to gravity for neutral hydrogen, varies from 0.9 at solar minimum to 1.5 at solar maximum (Woods et al. 2000). For $\mathrm{H}$, the major source of ionization is $\mathrm{CX}$ of $\mathrm{H}$ with solar wind protons, followed by EUV photo-ionization. Ionization rates $\beta_{i}$ as a function of heliographic latitude are derived from the SOHO-SWAN preliminary data analysis (Quémerais et al. 2006). During solar minimum $\beta_{i}$ is $6.6 \times 10^{-7} \mathrm{~s}^{-1}$ at heliographic latitudes between $\pm 20^{\circ}$ and $4 \times 10^{-7} \mathrm{~s}^{-1}$ at latitudes over $\pm 20^{\circ}$ and up to the poles. During solar maximum, the anisotropy is less important. It is scaled every $10^{\circ}$ with $\beta_{i}$ values between $8.4 \times 10^{-7} \mathrm{~s}^{-1}$ at the equator and $6.7 \times 10^{-7} \mathrm{~s}^{-1}$ at the poles.

For helium atoms $\mu=0$. Radiation pressure is negligible compared to solar gravitational attraction and the atoms are gravitationally focused downwind. The main cause of ionization is solar EUV radiation and electron impacts. The mean lifetime (the inverse of $\beta_{i}$ ) at $1 \mathrm{AU}$ varies from $1.4 \times 10^{7} \mathrm{~s}$ at solar minimum to $0.62 \times 10^{7} \mathrm{~s}$ at solar maximum, in agreement with McMullin et al. (2004).

Recent work has shown strong evidence for an anisotropic distribution of the He $30.4 \mathrm{~nm}$ solar irradiance and thus of the He photo-ionization rate (Witte et al. 2004; Auchère et al. 2005) and it has been shown that the electron impact ionization rate is also anisotropic (McMullin et al. 2004). However, since our model does not include any latitudinal dependence of the helium ionization rate, we use isotropic photo-ionization and electron impact ionization.

The radial dependence of electron impact ionization is taken from Rucinski \& Fahr (1989). Lallement et al. (2004) used it in an analysis of SOHO-UVCS $58.4 \mathrm{~nm}$ data, and found that the rate is appropriate for solar minimum, but requires a three-fold increase for solar maximum. In order to have self-consistency we use both the Rucinski \& Fahr (1989) radial dependence and the Lallement et al. (2004) electron impact ionization. Further 
Table 1. Slow and Fast Solar Wind Parameters. Cross sections for CX between SW heavy ions and H and He are based on theoretical and experimental work of (Beijers et al. 1994; Bliman et al. 1992; Bonnet et al. 1985; Dijkkamp et al. 1985a,b; Fritsch et al. 1996; Greenwood et al. 2001; Harel et al. 1992, 1998; Ishii et al. 2004; Iwai et al. 1982; Lee et al. 2004; Liu et al. 2005; Phaneuf et al. 1987; Richter et al. 1993; Shimakura et al. 1992; Suraud et al. 1991; Wu et al. 1994).

\begin{tabular}{|c|c|c|c|c|c|c|c|}
\hline \multicolumn{2}{|c|}{ SW type } & \multicolumn{3}{|c|}{ Slow } & \multicolumn{3}{|c|}{ Fast } \\
\hline $\begin{array}{l}V_{\mathrm{SW}}(\mathrm{k} \\
{\left[\frac{\mathrm{O}}{\mathrm{H}^{+}}\right]} \\
n_{\mathrm{H}^{+} \mathrm{o}} \text { at }\end{array}$ & $\begin{array}{l}\left.\mathrm{s}^{-1}\right) \\
\mathrm{AU}\left(\mathrm{cm}^{-3}\right)\end{array}$ & & $\begin{array}{c}400 \\
1 / 1780 \\
6.5\end{array}$ & & & $\begin{array}{c}750 \\
1 / 1550 \\
3.2\end{array}$ & \\
\hline $\mathrm{X}^{Q+}$ & $\begin{array}{l}\text { Spectral Lines }(\mathrm{keV})^{a} \\
\text { Produced by } \mathrm{X}^{*}(Q-1)+\end{array}$ & {$\left[\frac{\mathrm{X}^{Q+}}{\mathrm{O}}\right]^{b}$} & $\begin{array}{c}\sigma_{\left(\mathrm{H}, \mathrm{X}^{\mathrm{Q}^{+}}\right.} \\
\left(10^{-15} \mathrm{~cm}^{2}\right)\end{array}$ & $\begin{array}{c}\left.\sigma_{\left(\mathrm{He}, X^{Q+}\right.}\right) \\
\left(10^{-15} \mathrm{~cm}^{2}\right)\end{array}$ & {$\left[\frac{\mathrm{X}^{Q+}}{\mathrm{O}}\right]^{b}$} & $\begin{array}{c}\sigma_{\left(H, \mathrm{X}^{Q+}\right)} \\
\left(10^{-15} \mathrm{~cm}^{2}\right)\end{array}$ & $\begin{array}{c}\sigma_{\left(\mathrm{He}, X{ }^{{ }^{+}}\right)} \\
\left(10^{-15} \mathrm{~cm}^{2}\right)\end{array}$ \\
\hline $\mathrm{C}^{6+}$ & $(0.37,0.44,0.46)$ & 0.318 & 4.16 & 1.50 & 0.085 & 4.63 & 1.50 \\
\hline$C^{5+}$ & $(0.3,0.35,0.37)$ & 0.210 & 2.00 & 1.40 & 0.440 & 2.90 & 1.11 \\
\hline $\mathrm{N}^{7+}$ & $(0.25,0.5,0.6,0.62,0.64)$ & 0.006 & 5.67 & 2.00 & 0.000 & 5.55 & 2.00 \\
\hline $\mathrm{N}^{6+}$ & $(0.42,0.43,0.5)$ & 0.058 & 3.71 & 1.26 & 0.011 & 3.32 & 1.49 \\
\hline $\mathrm{N}^{5+}$ & $(0.05,0.059,0.065)$ & 0.065 & 2.27 & 1.41 & 0.127 & 2.92 & 1.07 \\
\hline $\mathrm{O}^{8+}$ & $(0.33,0.65,0.77,0.82,0.84)$ & 0.070 & 5.65 & 2.80 & 0.000 & 6.16 & 2.80 \\
\hline $\mathrm{O}^{7+}$ & $(0.561,0.569,0.574)$ & 0.200 & 3.40 & 1.80 & 0.030 & 3.70 & 1.97 \\
\hline $\mathrm{O}^{6+}$ & $(0.072,0.083,0.094,0.107)$ & 0.730 & 3.67 & 0.96 & 0.970 & 3.91 & 1.31 \\
\hline $\mathrm{Ne}^{9+}$ & $(0.905,0.915,0.922)$ & $0.030^{c}$ & 7.20 & 2.40 & $0.006^{c}$ & 7.20 & 2.40 \\
\hline $\mathrm{Ne}^{8+}$ & $(0.126,0.141,0.187)$ & 0.084 & 3.70 & 1.30 & 0.102 & 3.00 & 1.10 \\
\hline $\mathrm{Mg}^{11+}$ & $(1.33,1.34,1.35,1.37)$ & $0.035^{c}$ & 7.5 & 2.6 & $0.001^{c}$ & 7.5 & 2.6 \\
\hline $\mathrm{Mg}^{10+}$ & $(0.28,0.29,0.3,0.31)$ & 0.098 & 3.73 & 1.00 & 0.029 & 2.50 & 0.90 \\
\hline
\end{tabular}

${ }^{a}$ Including most important lines, ${ }^{b}$ Schwadron \& Cravens (2000), ${ }^{c}$ Rough estimate from ACE data.

work is, however, certainly needed to improve the accuracy of the helium ionization rate of helium (McMullin et al. 2004).

\subsection{Heavy solar wind ion distributions}

In the inner heliosphere and in the absence of charge transfer, the density of heavy ions would follow a $r^{-2}$ dependence. Here, in addition to the radial expansion, we consider the effect of collisions with interplanetary neutrals. The next step in our model is the computation of the heavy ion losses due to CX with hydrogen and helium atoms and the determination of a density distribution for each ion species. The process may be written:

$\mathrm{X}^{Q+}+[\mathrm{H}, \mathrm{He}] \rightarrow \mathrm{X}^{*(Q-1)+}+\left[\mathrm{H}^{+}, \mathrm{He}^{+}\right]$

and it represents the loss of ion $\mathrm{X}^{Q+}$ and the production of ion $\mathrm{X}^{(Q-1)+}$. The density of ion $\mathrm{X}^{Q+}$ is given by the equation:

$$
\begin{aligned}
\frac{\mathrm{d} N_{\mathrm{X}^{Q+}}}{\mathrm{d} x}= & -N_{\mathrm{X}^{Q+}}\left(\sigma_{\left(\mathrm{H}, \mathrm{X}^{Q+}\right)} n_{\mathrm{H}}(x)+\sigma_{\left(\mathrm{He}, \mathrm{X}^{Q+}\right)} n_{\mathrm{He}}(x)\right) \\
& +N_{\mathrm{X}^{(Q+1)+}}\left(\sigma_{\left(\mathrm{H}, \mathrm{X}^{(Q+1)+}\right)} n_{\mathrm{H}}(x)+\sigma_{\left(\mathrm{He}, \mathrm{X}^{(Q+1)+}\right)} n_{\mathrm{He}}(x)\right)
\end{aligned}
$$

where $x$ is the distance along the SW stream lines, $\sigma_{\left(\mathrm{H}, \mathrm{X}^{Q+}\right)}$ and $\sigma_{\left(\mathrm{He}, X^{Q+}\right)}$ are the hydrogen and helium CX cross-sections and $n_{\mathrm{H}}(x)$ and $n_{\mathrm{He}}(x)$ are the hydrogen and helium densities respectively. The first term represents the loss due to $\mathrm{CX}$ between $\mathrm{X}^{Q+}$ and $\mathrm{H}$ and $\mathrm{He}$ while the second term is the source term due to the equivalent $\mathrm{CX}$ process for $\mathrm{X}^{(Q+1)+}$. For bare ions $\left(\mathrm{C}^{6+}, \mathrm{N}^{7+}, \mathrm{O}^{8+}\right)$ the source term is, of course, absent, and in a first approximation we neglect it also for the lower state ions. We will discuss its role and justify this assumption in Sect. 3.3.

If we neglect this term, the ion density as a function of distance from the Sun is described by the equation:

$$
\begin{aligned}
& N_{\mathrm{X}^{Q+}}(r)= \\
& \quad \frac{N_{\mathrm{X}^{Q+} \mathrm{o}}}{r^{2}} \exp \left(-\int_{r_{\mathrm{o}}}^{r}\left(\sigma_{\left(\mathrm{H}, X^{Q+}\right)} n_{\mathrm{H}}(x)+\sigma_{\left(\mathrm{He}, \mathrm{X}^{Q+}\right)} n_{\mathrm{He}}(x)\right) \mathrm{d} x\right)
\end{aligned}
$$

where $r$ is the radial distance from the Sun and the density of ion $\mathrm{X}^{Q+}$ at $1 \mathrm{AU}$ is expressed in the form $N_{\mathrm{X}^{Q+}}=\left[\frac{\mathrm{X}^{Q+}}{\mathrm{O}}\right]\left[\frac{\mathrm{O}}{\mathrm{H}^{+}}\right] n_{\mathrm{H}^{+} \mathrm{o}}$, [O] being the total oxygen ion content of the solar wind and $n_{\mathrm{H}^{+} \mathrm{O}}$ the proton density at $1 \mathrm{AU}$. The adopted values of $\left[\frac{\mathrm{X}^{Q+}}{\mathrm{O}}\right]$ and $\left[\frac{\mathrm{O}}{\mathrm{H}^{+}}\right]$ for the fast and slow solar winds are given in Table 1 .

We assume that the heavy ion flux is propagating radially from the Sun. For each grid cell $(r, \lambda, \beta)$, where $\lambda$ and $\beta$ are the ecliptic coordinates, varying $1^{\circ}$ by $1^{\circ}$, the corresponding heliographic coordinates $\lambda_{\text {Helio }}$ and $\beta_{\text {Helio }}$ are calculated, and $\beta_{\text {Helio }}$ is used to discriminate between slow and fast solar wind.

During solar minimum we adopt the Slow Solar Wind composition (SSW) at an average velocity of $400 \mathrm{~km} \mathrm{~s}^{-1}$, accelerated in the equatorial zone $\left(\beta_{\text {Helio }}=\left[-20^{\circ}, 20^{\circ}\right]\right)$, and the Fast Solar Wind (FSW) composition with an average velocity of $750 \mathrm{~km} \mathrm{~s}^{-1}$, accelerated at higher heliographic latitudes $\left(\beta_{\text {Helio }}=\right.$ $\left.\left[-20^{\circ},-90^{\circ}\right],\left[20^{\circ}, 90^{\circ}\right]\right)$. The proton density $n_{\mathrm{H}]^{+} \mathrm{o}}$ is $6.5 \mathrm{~cm}^{-3}$ for the SSW and $3.2 \mathrm{~cm}^{-3}$ for the FSW. During solar maximum we assume that all the solar wind is in the SSW state. These values are consistent with the ionization rates we have used for the computation of the neutral densities. In Table 1 we summarize the parameters characterizing the two different states of the Solar Wind, as well as abundances $\left[\frac{\mathrm{X}^{Q+}}{\mathrm{O}}\right]$ and $\mathrm{CX}$ cross sections with $\mathrm{H}$ and $\mathrm{He}$ for each heavy ion species (and charge state) used in our analysis. The charge and elemental abundances of heavy ions in the slow and fast solar wind are adopted from Schwadron $\&$ Cravens (2000). We have updated values of cross sections of ion collisions with $\mathrm{H}$ and $\mathrm{He}$ atoms. The selective and total cross sections of the charge transfer collisions of the heavy solar wind ions have been constructed using relevant data from the laboratory measurements and theoretical calculations reported in Table 1.

We have calculated the number of $\mathrm{X}^{Q+}$ ions lost due to $\mathrm{CX}$ collisions with $\mathrm{H}$ and $\mathrm{He}$ atoms for the SW plasma flux propagating in the radial direction. For that we use a refined grid such that each grid cell is smaller than the $\mathrm{H}$ and He grid cells at each distance from the Sun so that we can obtain the best resolution for the affected regions of both neutral species. 


\subsection{Calculating the $X$-ray emission}

The final step of our simulation is the computation of the directional intensity $I(\lambda, \beta)$ of X-ray emission lines, resulting from CX collisions of the heavy SW ions $\mathrm{X}^{Q+}$ with the heliospheric $\mathrm{H}$ and $\mathrm{He}$ atoms. We compute the line of sight intensity $I$, seen by an observer at Earth orbit at different dates. CX collisions, producing X-ray photons, are described by the equation:

$$
\begin{aligned}
\mathrm{X}^{Q+}+[\mathrm{H}, \mathrm{He}] & \rightarrow \mathrm{X}^{*(Q-1)+}+\left[\mathrm{H}^{+}, \mathrm{He}^{+}\right] \\
& \rightarrow \mathrm{X}^{(Q-1)+}+\left[\mathrm{H}^{+}, \mathrm{He}^{+}\right]+\left[Y_{\left(E_{i}, \mathrm{H}\right)}, Y_{\left(E_{i}, \mathrm{He}\right)}\right]
\end{aligned}
$$

where $Y_{\left(E_{i}, \mathrm{H}\right)}, Y_{\left(E_{i}, \mathrm{He}\right)}$ is the photon yield for the spectral line $E_{i}$ induced in the CX between the ion $\mathrm{X}^{Q+}$ with $\mathrm{H}$ and He respectively. The values of quantum yields in collisions of the most important solar wind ions have been presented in articles on the charge transfer mechanism of cometary X-ray emission (Kharchenko \& Dalgarno 2000, 2001; Rigazio et al. 2002; Kharchenko et al. 2003; Pepino et al. 2004). The photon energies $E_{i}$ and relative intensities of different emission lines of the CX spectra have been computed for the fast and slow winds.

The volume collision frequency $R_{\mathrm{X}^{Q+}}$ in units of $\mathrm{cm}^{-3} \mathrm{~s}^{-1}$ of $\mathrm{X}^{Q+}$ ions with the neutral heliospheric atoms is given by the equation:

$$
\begin{aligned}
R_{\mathrm{X}^{Q^{+}}}(r) & =N_{\mathrm{X}^{Q+} \mathrm{o}}(r) v_{\mathrm{rel}}\left(\sigma_{\left(\mathrm{H}, \mathrm{X}^{Q+}\right)} n_{\mathrm{H}}(r)+\sigma_{\left(\mathrm{He}, \mathrm{X}^{Q+}\right)} n_{\mathrm{He}}(r)\right) \\
& =R_{\left(\mathrm{X}^{Q+}, \mathrm{H}\right)}(r)+R_{\left(\mathrm{X}^{Q+}, \mathrm{He}\right)}(r) .
\end{aligned}
$$

If we consider only the region inside the termination shock, where solar wind ions are supersonic, then the relative velocity between the solar wind ions and the IS neutrals, $\boldsymbol{v}_{\text {rel }}=\boldsymbol{V}_{\mathrm{SW}}-\boldsymbol{v}_{\mathrm{n}}$ in Eq. (5), can be approximated by $V_{\mathrm{SW}}$, since the neutral velocity $v_{\mathrm{n}} \ll V_{\mathrm{SW}}$. With the exception of Sect. 3.4 all the results have been obtained with this assumption. In Sect. 3.4 we examine in detail the case of the outer Heliosphere (the heliosheath and heliotail) where the IS neutral velocity and ion thermal velocity are no longer negligible.

Thus, for the inner heliosphere, the number of $h v$-photons emitted per second from unit volume is given by the formula:

$\varepsilon_{h v}(r)=R_{\left(\mathrm{X}^{Q+}, \mathrm{H}\right)}(r) Y_{(h v, \mathrm{H})}+R_{\left(\mathrm{X}^{Q+}, \mathrm{He}\right)}(r) Y_{(E h v, \mathrm{He})}$.

The intensity measured at energy $h v$ for an observer in position $\boldsymbol{O}\left(\lambda_{E}, \beta_{E}\right)$ for a line of $\operatorname{sight} \mathbf{L O S}(\lambda, \beta)$ is

$I_{h v}(\boldsymbol{O}, \mathbf{L O S})=\frac{1}{4 \pi} \int_{0}^{100 \mathrm{AU}} \varepsilon_{h v}(r) \mathrm{d} s$.

During solar maximum, the computation of the intensity is quite simple since we consider only one state of the Solar Wind, the slow one. This is not the case during solar minimum and especially for LOS towards high latitudes. In Fig. 9 (Supporting Online Material, hereafter SOM) we present a simplified observation geometry for such lines of sight for solar minimum conditions. In the figure, we have considered the Solar Equator parallel to the ecliptic plane but in reality the two planes have an angle of incidence of about $7.25^{\circ}$ which is taken into account in the modelling. As shown in Fig. 9 (SOM), a high latitude LOS crosses regions of both slow and fast SW and thus, the total intensity is a mixture of SSW and FSW induced photons. This is taken into account in our simulation when we interpolate in our heavy-ion grid for each point on the LOS according to its distance and latitude.

\section{Charge transfer EUV and X-ray maps of the heliosphere}

\subsection{Emissivity maps}

Figure 10 (SOM) shows the contour maps of the total emissivity of excited $\mathrm{O}^{* 5+}$ and $\mathrm{O}^{* 6+}$ ions produced in the charge transfer collisions of the SW ions with the hydrogen and helium gas during solar minimum. The top panels present iso-emissivity contours at positions $(x, y)$ between $\pm 15 \mathrm{AU}$ in the plane defined by the IS He wind axis and the vector $\left(\lambda=-16^{\circ}, \beta=0^{\circ}\right)$ which is very close to the ecliptic plane and, thus, contains mainly slow SW. In these figures, the IS wind is travelling from left to right. We recognize on the upwind side the crescent shape due to the $r^{-2}$ dependence of the solar wind flux and the spatial distribution of hydrogen. On the downwind side we find the excess of emissivity due to the focusing of He neutrals in the He cone.

The bottom panels of Fig. 10 (SOM) present iso-emissivity contours at positions $(x, z)$ between $\pm 15 \mathrm{AU}$ in the plane defined by the IS He wind axis $\left(\lambda=74^{\circ}, \beta=-5^{\circ}\right)$ and the heliospheric polar axis. The IS wind comes from the left and heads on $-5^{\circ}$ downwards with respect to the $z$ axis. The two panels stress the discontinuity at $\pm 20^{\circ}$ between the SSW and the FSW during solar minimum, as well as the contrast between the ions characterizing the two SW types. The discontinuity boundaries appear very sharp because we consider a simplified anisotropic model for the solar wind. The left panels present iso-emissivity contours for the $\mathrm{O}^{5+}$ line at $0.08 \mathrm{keV}$ (sum of the lines 0.072 and $0.082 \mathrm{keV}$ ), generated from $\mathrm{O}^{6+}$, abundant in the fast $\mathrm{SW}$. The right column presents contours for the sum of intensities of $\mathrm{O}^{* 6+}$ emission lines of $0.561,0.569$ and $0.574 \mathrm{keV}$, induced in radiative transitions from the triplet and singlet excited states of $\mathrm{O}^{* 6+}$. We show in Fig. 10 (SOM) the average energy of $\mathrm{O}^{* 6+}$ photons: $0.57 \mathrm{keV}$. The $\mathrm{O}^{7+} \mathrm{SW}$ ions, producing excited $\mathrm{O}^{* 6+}$, are more abundant in the SSW.

\subsection{Intensity maps}

In Figs. 1 (and 11, 12, 13, SOM) we present full-sky maps of the heliospheric X-ray emission in ecliptic coordinates. Color in these figures represents intensities of the $\mathrm{CX}$ emission given in $10^{-9} \mathrm{erg} \mathrm{cm}^{-2} \mathrm{sr}^{-1} \mathrm{~s}^{-1}$, the red colour corresponding to minimum and the blue to maximum values. We also mark in each map contours at $10 \%$ (white), 50\% (grey) and 90\% (black) of maximum value. We have removed from every map a data portion of $20^{\circ} \times 20^{\circ}$ around the solar disk where no instrument can observe. In the following we examine the different effects illustrated in these maps.

\subsubsection{Solar cycle effects}

Again, in Figs. 1 and 11, 12, 13 (SOM) we chose two oxygen ions characterizing the slow or the fast $\mathrm{SW}$, in order to emphasize the contrast between the two SW states. In the two top panels of each column we illustrate the $\mathrm{O}^{7+} 0.65 \mathrm{keV}$ line for solar maximum and solar minimum conditions. In the third and fourth panel of each column we present the $\mathrm{O}^{5+} 0.08 \mathrm{keV}$ line for solar maximum and solar minimum respectively.

The striking features are: (i) at solar minimum, the modeled intensity in the equatorial region is conspicuously different from regions at medium or high latitudes. This is entirely due to the solar wind latitudinal structures, separating the slow from the fast solar wind, shown in Fig. 9 (SOM). For the fast solar wind, the major contributors to the $\mathrm{CX}$ emission are low charge such 
Fig. 1. Solar maximum and solar minimum full sky monochromatic for the $\mathrm{O}^{7+}$ line at $0.65 \mathrm{keV}$ and the $\mathrm{O}^{5+}$ line at $0.08 \mathrm{keV}$. The observer is situated at $75^{\circ}$ (left column) and at $251^{\circ}$ (right column) ecliptic longitude. The color scale is in units of $10^{-9} \mathrm{erg} \mathrm{cm}^{-2} \mathrm{sr}^{-1} \mathrm{~s}^{-1}$, red colour corresponding to minimum and blue to maximum values. The masked area is the $20^{\circ} \times 20^{\circ}$ region around the solar disk. The map is shown in ecliptic coordinates.

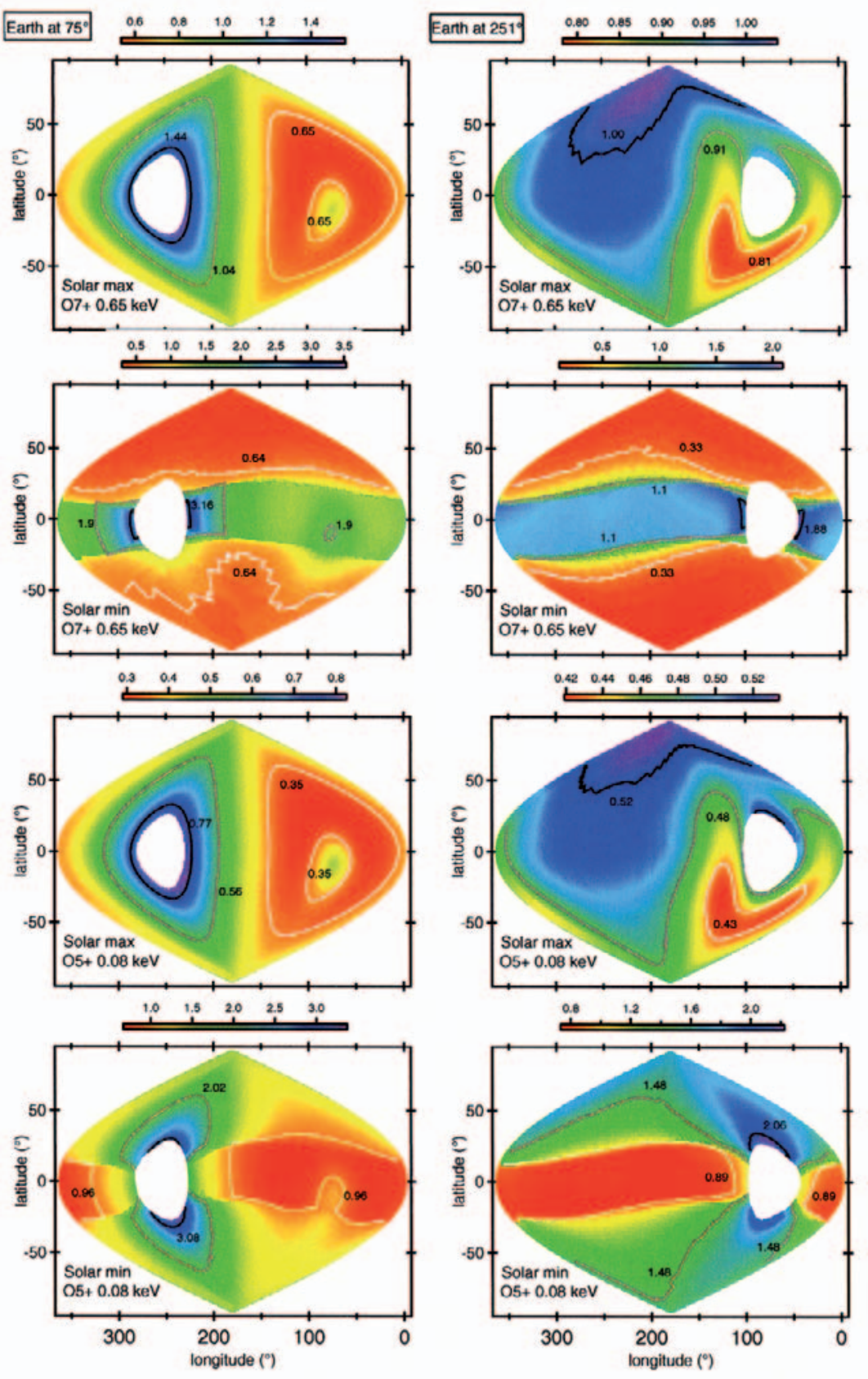

as $\mathrm{C}^{4+}, \mathrm{N}^{5+}$ and in particular $\mathrm{O}^{5+}$, illustrated in Fig. 1 . These ions emit in the EUV $(E \leq 150 \mathrm{eV})$. The slow solar wind is characterized by highly charged ions $\mathrm{C}^{6+}, \mathrm{N}^{7+}, \mathrm{O}^{8+}$ and $\mathrm{Ne}^{9+}$ which produce harder X-ray emitting ions, as for example $\mathrm{O}^{7+}$ illustrated in the maps.

(ii) The X-ray emission pattern for solar maximum is very similar for both $\mathrm{O}^{5+}$ and $\mathrm{O}^{7+}$ lines, but for solar minimum there are strong differences. While $\mathrm{O}^{7+}$ emission is almost absent at high ecliptic latitudes, $\mathrm{O}^{5+}$ is much brighter due to the difference in relative abundance of $\mathrm{O}^{8+}$ and $\mathrm{O}^{6+} \cdot \mathrm{O}^{7+}$ is generated during $\mathrm{CX}$ between $\mathrm{O}^{8+}$ ion and $\mathrm{H}$ and He. $\mathrm{O}^{8+}$ is absent from the fast solar wind. The $\mathrm{O}^{7+}$ emission is never zero, because there are no lines of sight containing only the FSW.

(iii) At low latitudes, near the solar equator, X-ray emissions are more intense for solar minimum than for solar maximum because the neutral atom content is higher during solar minimum.
During solar minimum, photo-ionization is less efficient and $\mathrm{H}$ and $\mathrm{He}$ are less readily destroyed by photo-ionization. Further, gravitational pressure exceeds radiation pressure and thus neutral $\mathrm{H}$ atoms have incoming trajectories and fill the ionization cavity.

\subsubsection{Effect of the observer's position}

Each column represents a different observing position on the Earth's orbit. We cover half an orbit, about every $45^{\circ}$ starting downwind $\left(\lambda_{E}=75^{\circ}\right.$, Fig. 1, left $)$ to upwind $\left(\lambda_{E}=251^{\circ}\right.$, Fig. 1, right). When examining the different vantage points on the Earth's orbit, it is no longer the solar wind ion distribution that is the source of the X-ray pattern differences, but the $\mathrm{H}$ and He distributions in interplanetary space. These differences are 
even more striking in the solar maximum maps, where only one solar wind type is present.

When the observer is situated downwind (see Fig. 1, left), emission is dominated by $\mathrm{CX}$ with He. Maximum intensity is observed for a ring of LOS upwind, around the forbidden solar direction. These LOS cross all the ionization cavity, extending to several $\mathrm{AU}$, before reaching the first $\mathrm{H}$ atoms. Thus the maximum in intensity is due to He concentrated around and behind the sun, on the downwind (DW) side. In this region the focusing cone $\left(\lambda=75^{\circ}, \beta=-5^{\circ}\right)$ produces an excess of $\mathrm{CX}$ and thus of $\mathrm{X}$-ray intensity. $\mathrm{H}$ atoms are absent downwind, where the ionization cavity limits the neutral atom abundance. This is illustrated by the minimum of emission in the LOS forming a large ring around the He cone.

These characteristic features evolve gradually when moving on the Earth's orbit from downwind to upwind (Figs. 11-13 SOM). Maximum emission is still located close around the sun but the emission pattern loses its axisymmetric form. The He cone becomes less and less striking, but still distinguishable by an excess of emission when observing a LOS that crosses it. The minimum X-ray intensity obtains a large oval-like pattern in LOS pointing downwind, less and less disturbed by the presence of the He cone as we move upwind.

On an upwind position of the observer (Fig. 1, right), the major source is $\mathrm{CX}$ with $\mathrm{H}$ with maximum $\mathrm{X}$-ray intensity on the northern antisolar lines of sight, towards the incoming flow. Those antisolar LOS cross a much smaller portion of the ionization cavity than LOS pointing downwind. They therefore see more $\mathrm{H}$ atoms approaching, while they miss $\mathrm{He}$ atoms which are absent at distances greater than $1 \mathrm{AU}$ from the sun. Minimum $\mathrm{X}$-ray intensity on these maps is thus situated in the southern hemisphere towards the Sun (DW) illustrating the major loss of $\mathrm{H}$ neutrals in the ionization cavity. Even though there is little $\mathrm{He}$ on the upwind maps, it is not completely absent and we still find a halo of X-ray emission around the Sun.

\subsection{The secondary ion charge transfer source term}

Secondary ions contribute to the total heliospheric emission. In Fig. 2 we show how the ratio of the total ion density, including secondary CX-produced ions (Eq. (2)), to the density calculated with the primary CX destruction term (Eq. (3)) only, varies with distance to the Sun. We show this ratio for two different ions originating from slow $\mathrm{SW}$ regions, $\mathrm{C}^{5+}$ and $\mathrm{O}^{7+}$, moving in two radial directions, and heading upwind or downwind.

$\mathrm{C}^{5+}$ is produced in $\mathrm{C}^{6+}+\mathrm{H} \mathrm{CX}$ collisions with a high value of cross section and depleted in the $\mathrm{CX}^{5+}+\mathrm{H}$ and $\mathrm{C}^{5+}+$ $\mathrm{He}$ collisions, with cross sections that are significantly smaller. Moreover, $\mathrm{C}^{6+}$ is more abundant than $\mathrm{C}^{5+}$ in the slow SW, and $\mathrm{CX}$ of $\mathrm{C}^{6+}$ is a large source of $\mathrm{C}^{5+}$. The gain of $\mathrm{C}^{5+}$ exceeds the loss and its density increases rapidly at large distances from the Sun. For the other ions the source term is less important but it slows the decrease that would occur in its absence. The ion creation term becomes effective at large distances from the Sun of 30-40 AU, beyond the region where most of the emission takes place. For an upwind LOS, $88 \%$ of the total $\mathrm{X}$-ray emission due to $\mathrm{H}$ is produced in the first $10 \mathrm{AU}$ and $98 \%$ of the total $\mathrm{X}$-ray emission due to $\mathrm{He}$ is produced within the first $5 \mathrm{AU}$. We conclude that the secondary source term does not add more than $4 \%$ to the total X-ray emission of the inner Heliosphere, and therefore we can neglect it in a first approach.

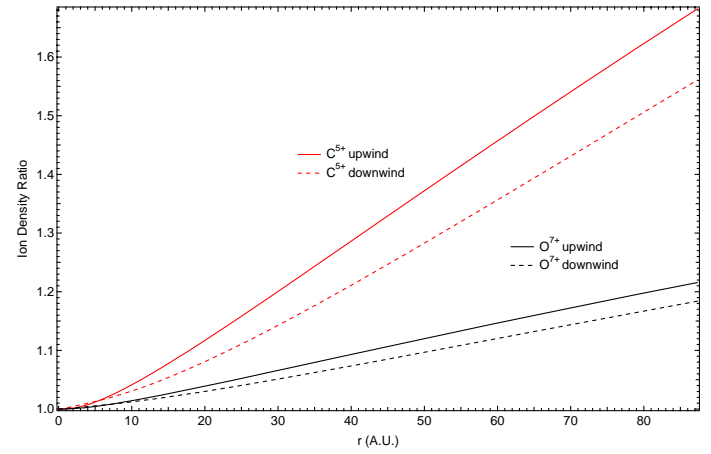

Fig. 2. Ratio between the total ion density (including secondary CX sources) and primary ion density as a function of distance from the Sun.

\subsection{The outer heliosphere contribution}

In the previous sections, we analyzed the various effects of the solar cycle and the observer's position on the total emission maps. The maps refer to the inner heliosphere, where the solar wind is supersonic and flows radially. Beyond the termination shock it is forced to decelerate and turn backwards at the heliopause. The relative contribution of the inner heliosheath and the heliotail to the total X-ray emission has been computed separately using the plasma and neutral distributions of Izmodenov \& Alexashov (2003). In this kinetic-continuum model (Baranov \& Malama 1993), the solar wind at the orbit of the Earth is steady and spherically symmetric. The interstellar flow which consists of hydrogen atoms is uniform and plane-parallel outside the heliosphere. In these conditions the flow is steady and axisymmetric. All charged particles (electrons, protons, alpha-particles, pickup ions) are treated as a single component fluid, described by the hydrodynamic Euler equations with source terms that include the effects of CX. The motion of the interstellar atoms in the heliospheric interface is determined by solving the kinetic equation. It is assumed in the model that $\mathrm{H}$ atoms interact with the plasma by CX only and electron impact is neglected. Computations have been carried out far in the tail of the interaction region. This self-consistent neutral-plasma model yields $\mathrm{H}$ and ion densities, thermal velocities and flow velocities along streamlines. We use these results out to $3000 \mathrm{AU}$ beyond which the production of $\mathrm{X}$ rays is negligible.

In Fig. 3 are shown the solar wind streamlines from the self consistent plasma/neutrals model. The IS wind flows from right to left pushing the solar wind plasma and creating the heliospheric interface. In a first step, ion losses have been computed along each solar wind streamline. Up to the termination shock the loss term has been calculated as described earlier. Once the termination shock is crossed, we can no longer neglect the thermal motion of the ions which becomes important in the heliosheath, and in Eq. (5) we replace $V_{\mathrm{SW}}$ by $v_{\text {rel, } H S}$ where

$v_{\mathrm{rel}, H S}=\sqrt{\left(v_{\mathrm{rel}}^{2}+8 k T_{i} / \pi m_{i}\right)}$

includes the relative velocity between ions and neutrals $\boldsymbol{v}_{\text {rel }}=$ $\boldsymbol{V}_{\mathrm{SW}}-\boldsymbol{v}_{\mathrm{n}}$ and the ion thermal motion.

Except for the upwind and downwind direction, any line of sight in the heliosheath crosses all the streamlines making at the Sun an angle between 0 and 90 degrees. Since ions displaced from the forward direction continue to exchange with the neutral atoms the computation of the intensities makes use of the loss along the streamlines. For the upwind LOS emission ceases at the heliopause. For the downwind LOS ions contribute to the 


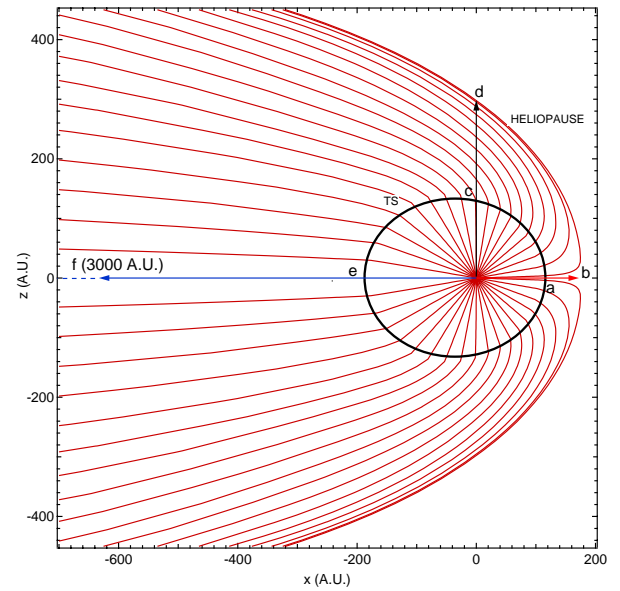

Fig. 3. Solar wind streamlines calculated with the Izmodenov \& Alexashov (2003) model. Arrows mark the three LOS for which the contribution of the outer heliosphere has been calculated and presented in Fig. 4.

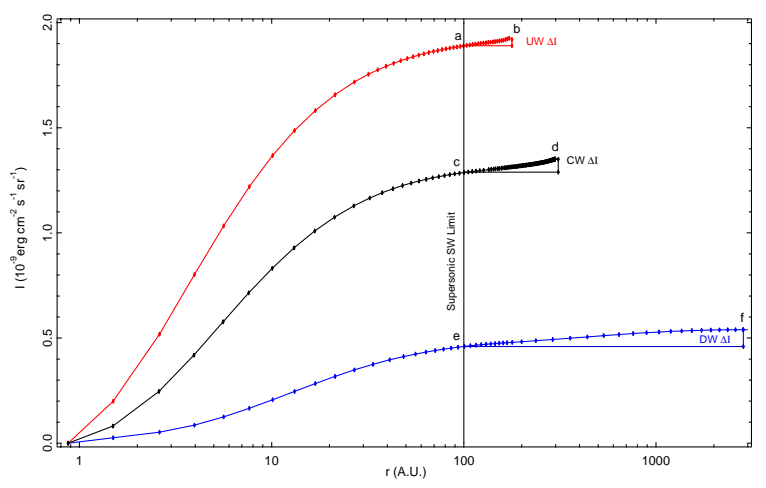

Fig. 4. Contribution of the heliosheath upwind (red) and crosswind (black), and the heliotail (blue) to a heavy SW ion emission. Intensity, in units of $10^{-9} \mathrm{erg} \mathrm{cm}^{-2} \mathrm{~s}^{-1} \mathrm{sr}^{-1}$, is presented as a function of distance $r$ to the Sun (in AU). See location of (a) to (f) points in Fig. 3.

emission at large distances out to $3000 \mathrm{AU}$ where the ions have all been removed by $\mathrm{CX}$.

In the heliosheath, CX with atomic hydrogen dominates. Gravitational focusing and ionization effects which both enhance the concentration of He over $\mathrm{H}$ close to the Sun are not at work at large distances and in estimating the X-rays from the heliosheath we may ignore He. We may also ignore solar activity which is limited in its effects at large distances and consider the intensities produced in a stationary slow solar wind. In Fig. 4, we present the total emission in units of erg $\mathrm{cm}^{-2} \mathrm{~s}^{-1} \mathrm{sr}^{-1}$ from CX with $\mathrm{H}$ as a function of distance in $\mathrm{AU}$ from the Sun on three LOS, upwind, crosswind and downwind, for a hypothetical ion of atomic number 16 with a cross-section of $3.5 \times 10^{-15} \mathrm{~cm}^{2}$ and at a spectral line of $0.56 \mathrm{keV}$.

In Table 2 we present the contributions of emissions in the outer heliosphere from individual ions, expressed as percentages of the total emission intensity due to $\mathrm{H}$ and total emission due to $\mathrm{H}+\mathrm{He}$, received within $100 \mathrm{AU}$. The corresponding amount is much larger downwind and depends essentially on the cross section of $\mathrm{CX}$ with $\mathrm{H}$ atoms, decreasing as the cross section increases. The largest enhancement happens for $\mathrm{C}^{5+}$ which has the smallest cross section and is depleted less rapidly with distance. Upwind, the heliosheath extends to about 170 AU and its contribution is small at $2.7 \%$ of the total. Crosswind there is a small
Table 2. Contribution of the Outer Heliosphere, up to $\sim 170$ AU UW, $\sim 300 \mathrm{AU}$ CW and $\sim 3000 \mathrm{AU} \mathrm{DW}$, to the X-ray intensity due to CX between heavy SW ions and IS $\mathrm{H}$ atoms and to the total directional $\mathrm{X}$-ray intensity $(\mathrm{H}+\mathrm{He})$ within $100 \mathrm{AU}$.

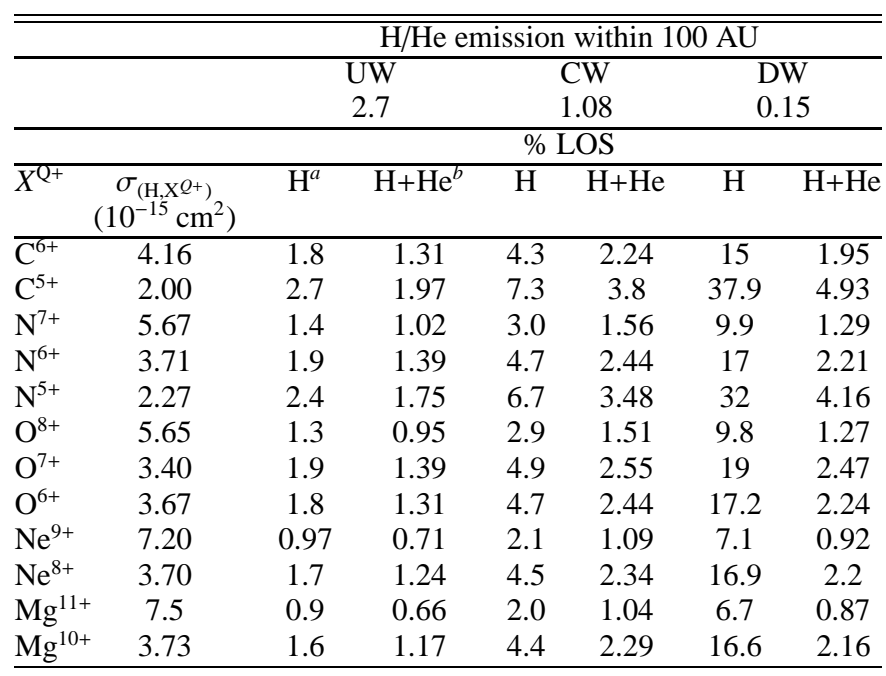

${ }^{a} \%$ to add to the emission due to $\mathrm{CX}$ with $\mathrm{H} ;{ }^{b} \%$ to add to the total emission due to $\mathrm{CX}$ with $\mathrm{H}$ and $\mathrm{He}$.

increase to $7.3 \%$, the heliopause occuring at 300 AU. A substantial enhancement of $38 \%$ results for the downwind direction because CX contributes out to $3000 \mathrm{AU}$.

As stated above, in the external heliosphere most of the emission comes from hydrogen. In Table 2 we show the resulting contributions to the emission generated by collisions with $\mathrm{H}$ and the resulting contribution to the total emission. The contributions of the heliosheath to the total intensities are smaller on the downwind hemisphere since there, $\mathrm{CX}$ with $\mathrm{He}$ is the dominant source of emission. Globally, due to this counteracting effect, the contribution is limited to about $5 \%$.

\section{EUV and soft X-ray spectra}

EUV and soft X-ray spectra for three LOS from two locations of the observer at solar minimum and solar maximum are presented in Figs. 5 and 6.

The LOS are specified by $\beta_{\mathrm{LOS}}=-90^{\circ}$, South heliospheric Pole LOS, $\beta_{\mathrm{LOS}}=90^{\circ}$, the North heliospheric Pole and $\beta_{\mathrm{LOS}}=$ $0^{\circ}$, in the equatorial plane. The LOS with $\beta_{\mathrm{LOS}}=0^{\circ}$ is oriented towards the anti-solar direction for each position of the observer. Thus, when the observer is located upwind at $\lambda_{E}=251^{\circ}$ the equatorial LOS is directed towards the incoming neutral atom flow, $\lambda_{\mathrm{H}} \sim 252^{\circ}, \beta_{\mathrm{H}} \sim 8^{\circ}$. When the observer is located downwind at $\lambda_{E}=75^{\circ}$, the equatorial LOS is directed almost inside the Helium focusing cone $\left(\lambda_{\mathrm{He}}=74^{\circ}, \beta_{\mathrm{He}}=-5^{\circ}\right)$.

The spectra include the emission lines from the ions listed in Table 1 , at energies between $0.005 \mathrm{keV}$ to $1.4 \mathrm{keV}$. They exclude a small fraction of heavier ions $\mathrm{Fe}^{Q+}, \mathrm{Si}^{Q+}$ and $\mathrm{S}^{Q+}$ which produce lines in the $300 \mathrm{eV}$ energy range. Each line is a Gaussian profile with $F W H M$ of $12 \mathrm{eV}$, which corresponds to the resolution achieved by McGammon et al. (2002). The EUV and X-ray spectra arising from CX vary significantly with solar activity, line of sight and observer location. 

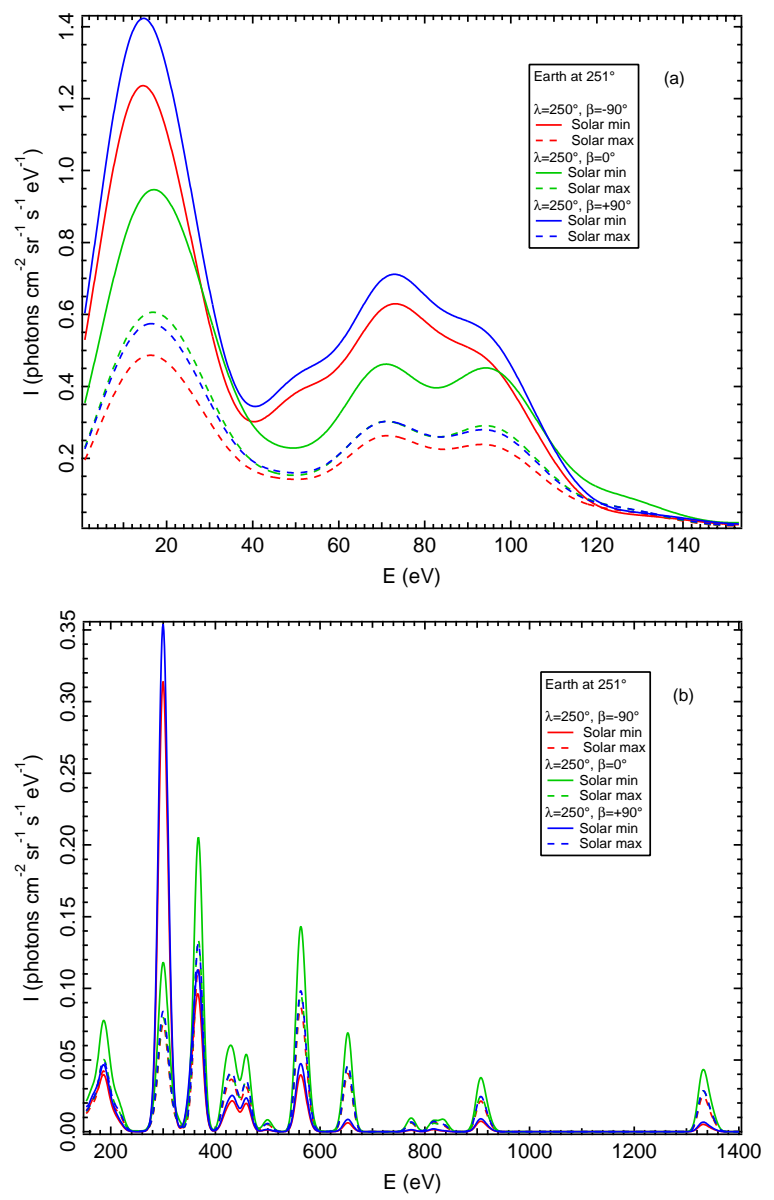

Fig. 5. Heliospheric EUV a) and X-ray b) emission spectra for an observer located upwind pointing in three different lines of sight: north ecliptic pole in blue, south ecliptic pole in red and equatorial antisolar LOS in green. Plain lines correspond to solar minimum conditions and dashed lines correspond to solar maximum conditions. Units are photons $\mathrm{cm}^{-2} \mathrm{~s}^{-1} \mathrm{sr}^{-1} \mathrm{eV}^{-1}$.

\section{Effect of an impulsive solar event on the X-ray emission temporal profile: the Hubble Deep Field-North observation}

SCK04 observed the HDFN with the XMM-Newton European Photon Imaging Camera (EPIC) on June 1, 2001 and presented a temporal and spectroscopic analysis of the diffuse X-ray emission in the range $E<1.5 \mathrm{keV}$. They identify in their observations the $\mathrm{X}$-ray spectrum expected from $\mathrm{CX}$ emission of the highly ionized solar wind ions, characterized by $\mathrm{C}$ VI lines at 0.37 and $0.46 \mathrm{keV}, \mathrm{O}$ VII line at $0.57 \mathrm{keV}$ (the triplet 0.561 , $0.568,0.574 \mathrm{keV}), \mathrm{O}$ VIII lines at 0.65 and $\sim 0.8 \mathrm{keV}$, Ne XI lines at $\sim 0.92 \mathrm{keV}$, and $\mathrm{Mg}$ XI lines at $\sim 1.35 \mathrm{keV}$. They observed a strong X-ray intensity enhancement in this energy range during one of the long exposures and they correlated the increase with an enhancement of both the solar wind flux and the highly ionized heavy ion abundances, in particular with a high $\mathrm{O}^{7+} / \mathrm{O}^{6+}$ ratio.

They considered the possible targets to be interstellar neutral $\mathrm{H}$ and $\mathrm{He}$ atoms or exospheric $\mathrm{H}$ atoms of the geocorona. Neither source appears to provide a satisfactory explanation of the temporal variation of the intensities. The emissions and the SW enhancement cut off at the same time, suggesting a terrestrial origin, yet the intensity remained nearly constant over a long
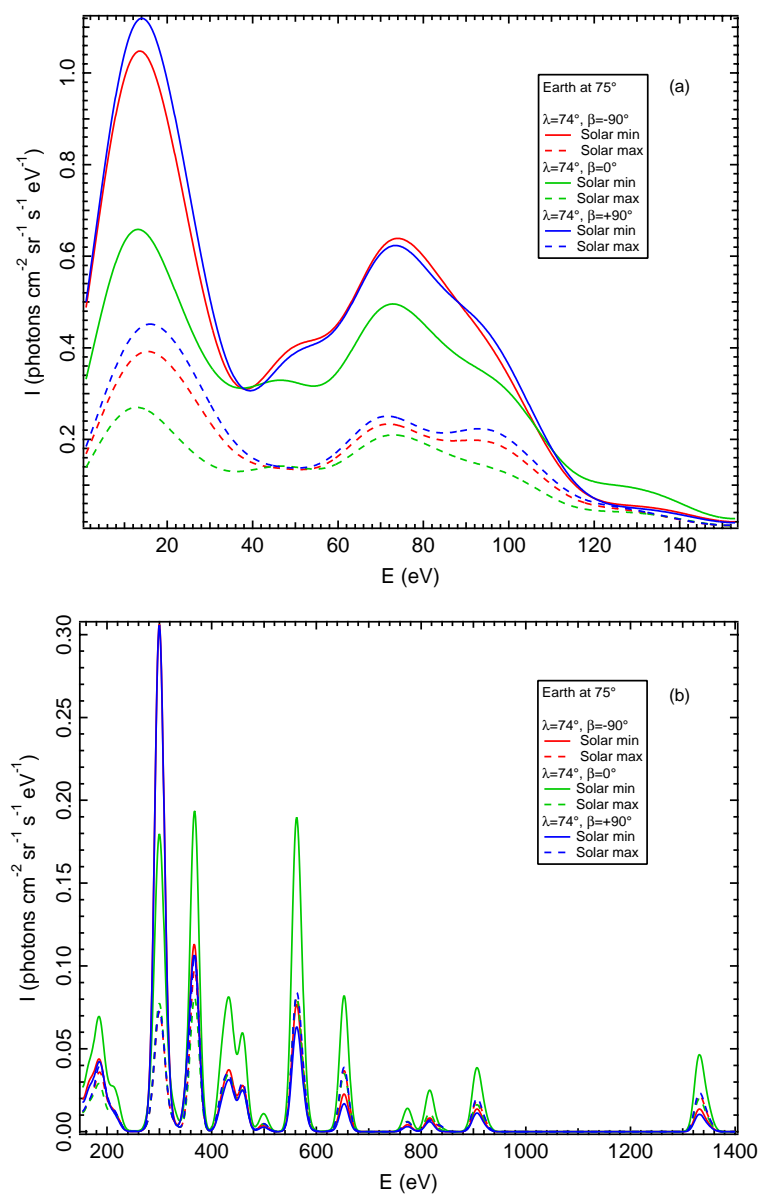

Fig. 6. Same as Fig. 5 but for an observer located downwind. Units are photons $\mathrm{cm}^{-2} \mathrm{~s}^{-1} \mathrm{sr}^{-1} \mathrm{eV}^{-1}$.

period of time during which the SW varied by a factor between 3 and 6, consistent with a distributed heliospheric emission.

We explore the temporal evolution of the X-ray emission detected along a given LOS in the interplanetary space, under the influence of a solar wind enhancement. We model the location of the solar wind as a function of time along a Parker-type spiral, taking account of solar rotation. We assume that the LOS is entirely contained within the solar equatorial plane and that the rotation axis is perpendicular to the ecliptic. The former assumption is questionable. However, we have made some simulation in similar geometries for a $45^{\circ}$ inclined LOS and for an active region extended in latitude, for which we obtain similar results. In addition, we are interested in the way the rotation influences the temporal behaviour of the emission, and how it is combined with the non-stationary ionization.

We consider two regimes of X-ray emission, high and low (Snowden et al. 2004). The observational parameters are given in Table 3. Observations began on June 1, 2001 at 08:16:36 UT, about $0.2 \mathrm{~d}$ before the SW flux enhancement. The observer was at $\lambda_{E}=251^{\circ}, \beta=0.0^{\circ}$ and the LOS along which XMM-Newton EPIC was pointing was $\lambda_{\mathrm{HDFN}}=148^{\circ}, \beta_{\mathrm{HDFN}}=+57^{\circ}$ corresponding to a heliographic latitude $\beta_{\text {helio }}=+50^{\circ}$. For the model the LOS is in the ecliptic plane, towards $\lambda=148^{\circ}$ and the observer is at $\lambda_{E}=251^{\circ}$ to match as well as possible the XMMNewton observation.

We define the proton flux in units of $2.6 \times 10^{8} \mathrm{~cm}^{-2} \mathrm{~s}^{-1}$ which is the typical SSW value for the quiet Sun at solar maximum. We call it the slow proton flux unit (SPFU). In our simulation the 
Table 3. Parameters of XMM-Newton observation of HDFN on June 1, 2001.

\begin{tabular}{|c|c|c|c|c|}
\hline \multicolumn{5}{|c|}{ Observation geometry parameters } \\
\hline \multicolumn{5}{|c|}{$\begin{array}{l}2001 \text { June } 01, \lambda_{\mathrm{XMM}}=251^{\circ} \\
\lambda_{\mathrm{HDFN}}=148^{\circ}, \beta_{\mathrm{HDFN}}=+57^{\circ}\end{array}$} \\
\hline Solar Wind Parameters & \multicolumn{2}{|c|}{ High } & \multicolumn{2}{|r|}{ Low } \\
\hline$\overline{\mathrm{H}}^{+}$flux SPFU ${ }^{a}$ & \multicolumn{2}{|c|}{3.8} & \multicolumn{2}{|r|}{1.15} \\
\hline \multirow[t]{3}{*}{$\mathrm{O}^{7+} / \mathrm{O}^{6+b}$} & \multicolumn{2}{|c|}{0.99} & \multicolumn{2}{|r|}{0.15} \\
\hline & \multicolumn{2}{|c|}{ Model $1^{b}$} & \multicolumn{2}{|r|}{ Model $2^{c}$} \\
\hline & High & Low & High & Low \\
\hline $\mathrm{O}^{8+} / \mathrm{O}^{7+}$ & $0.05::$ & $1::$ & 0.57 & 0.33 \\
\hline Line & \multicolumn{4}{|c|}{ Model Energy Flux $\left(10^{-9} \mathrm{erg} \mathrm{cm}^{-2} \mathrm{sr}^{-1} \mathrm{~s}^{-1}\right)$} \\
\hline O VII $0.57 \mathrm{keV}$ & 12.5 & 7.04 & 11.5 & 7.26 \\
\hline O VIII $0.65 \mathrm{keV}$ & 6.28 & 6.5 & 6.56 & 3.38 \\
\hline
\end{tabular}

${ }^{a}$ ACE SWEPAM data, SPFU $=2.610^{8} \mathrm{~cm}^{-2} \mathrm{~s}^{-1} ;{ }^{b}$ ACE SWICS data;

${ }^{c}$ For these choices, see Sect. 5.

base level is 1.15 SPFU derived from ACE/SWEPAM data also (SCK04). From the same data we represent the SW flux during the enhancement by a step function whose area is equal to the integral of the measured flux during the same period of time. This corresponds to a mean value of about 3.8 SPFU, a relative increase of a factor of 3.3. The high X-ray emission regime occurs before and during the SW enhancement and the low X-ray emission regime starts with the SW enhancement cut-off (SCK04).

According to $\mathrm{SCK} 04$ the $\mathrm{O}^{7+} / \mathrm{O}^{6+}$ ratio was significantly high at around 0.99 during the $\mathrm{X}$-ray high emission regime and dropped to 0.15 just when the SW flux measurements came back to normal values. SCK04 estimate that an equivalent enhancement should have occurred for the relative abundances of the ionization states of $\mathrm{O}^{8+}, \mathrm{Ne}^{9+}$ and $\mathrm{Mg}^{11+}$, but also cite contradictory observations from preliminary ACE results for the $\mathrm{O}^{8+} / \mathrm{O}^{7+}$ ratio. According to these data, the $\mathrm{O}^{8+} / \mathrm{O}^{7+}$ ratio was curiously low, about 0.05 , during the event and increased by a factor of 20-30 just after the event.

We have investigated these two situations, focusing on the two oxygen ions $\mathrm{O}^{8+}$ and $\mathrm{O}^{7+}$, which produce excited $\mathrm{O}^{7+}$ and $\mathrm{O}^{6+}$ ions respectively. Their de-excitation gives rise to the most intense lines in the energy range $0.52-0.75 \mathrm{keV}$ : the O VII line at $0.57 \mathrm{keV}$ (the triplet $0.561,0.568,0.575 \mathrm{keV}$ ) and the O VIII line at $0.65 \mathrm{keV}$, best detected by XMM.

We have simplified the description of the solar event by considering an active region (AR) with a longitudinal size of about $6^{\circ}$, rotating with the Sun (360 degrees per 27 days), and assumed it persists throughout a time of 5 days. If we assume that the active region is continually expelling material, the enhancement will be described as a step function during about half a day. The expelled material, propagating radially at $400 \mathrm{~km} \mathrm{~s}^{-1}$, travels for about $4.3 \mathrm{~d}$ before reaching the Earth while the AR turns through $57^{\circ}$ on the solar disk.

In Fig. 7 we illustrate the position of the SW enhancement on day 0 at the start of XMM-Newton observation. The satellite is at $\lambda=251^{\circ}$ with EPIC pointing towards $\lambda_{\mathrm{HDFN}}=148^{\circ} 0.2 \mathrm{~d}$ before the measurements of the SW enhancement. The sections of LOS that respond to the presence of the SW enhancement, at this precise moment, are represented by the intersections of the dark purple arm with the LOS. All parts of the LOS between the two intersections have been reached earlier at the times indicated by the corresponding colours.

When the SW flux, enhanced by a factor $E$, encounters the neutral atoms in interplanetary space, the X-ray emission intensity is increased proportionally. In parallel, because CX with

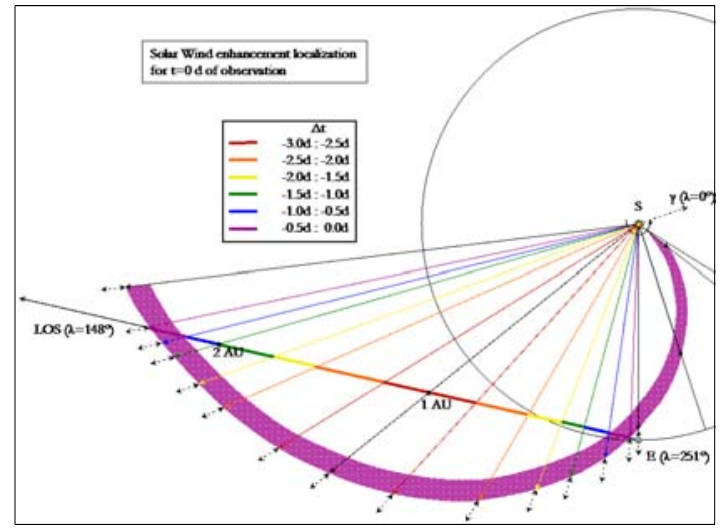

Fig. 7. Solar Wind enhancement localization for the start of the XMM HDFN X-ray observation.

protons is the main source of ionization of IS $\mathrm{H}$ atoms, the ionization rate is increased by the same factor $E$. The helium ionization rate is also slightly affected. The changes may be represented approximately by the ionization rate ratios $\beta_{\text {high }} / \beta_{\text {low }}=$ $(E+0.1) / 1.1$ for $\mathrm{H}$ and $\beta_{\text {high }} / \beta_{\text {low }}=(0.1 \cdot E+1) / 1.1$ for He. Therefore, we have a global decrease in the volumic density of both neutral species which is calculated in the model. The loss of emissivity due to this decrease of neutral density, though, is much fainter than the direct gain from the increased SW flux. Once the SW has passed through the region the neutral densities relax to lower equilibrium values with a modified SW ion distribution and a diminished X-ray intensity.

Along lines of sight, $88 \%$ of the total $\mathrm{X}$-ray emission due to $\mathrm{H}$ is produced in the first $10 \mathrm{AU}$ and $42 \%$ in the first $2 \mathrm{AU}$. For $\mathrm{He}, 98 \%$ of the total X-ray emission is produced within the first $5 \mathrm{AU}$ and $88 \%$ in the first $2 \mathrm{AU}$. Figure 7 shows that the major portion of the enhanced X-ray emission on the $\operatorname{LOS}\left(\lambda=148^{\circ}\right)$ is produced before the $\mathrm{SW}$ enhancement is registered by ACE and Wind solar wind instruments.

In Fig. 8 we present the results of simulations and SCK04 data. In the bottom panels we give the solar wind input parameters and in the upper panels the X-ray emissions derived from the simulations together with the mean values from the observational data (SCK04). The black dashed line shows the simulated SW flux enhancement in SPFU as it would be measured by an instrument at the position of the Earth $\left(\lambda=251^{\circ}, 1 \mathrm{AU}\right)$. The plain green vertical lines correspond to the start and end of XMM observations, while the dashed green vertical line delimits the high and low X-ray emission regimes, and the end of the SW enhancement. The crosshatched parts of the graphs represent the periods where no X-ray observations in this LOS are available. The red and blue dashed lines represent the $\mathrm{O}^{7+} / \mathrm{O}^{6+}$ and $\mathrm{O}^{8+} / \mathrm{O}^{7+}$ ratios respectively, associated with the variations of $\mathrm{SW}$ proton flux.

Before the SW enhancement, we adopt the ratios $\mathrm{O}^{7+} / \mathrm{O}^{6+}$ $=0.27$ and $\mathrm{O}^{8+} / \mathrm{O}^{7+}=0.35$ (Schwadron \& Cravens 2000). For the time during and after the SW enhancement we consider two possibilities. One, described in panels $1 \mathrm{a}$ and $1 \mathrm{~b}$, uses the ACE/SWICS data in the SCK04 analysis which showed a very high $\mathrm{O}^{7+} / \mathrm{O}^{6+}$ ratio during the enhancement, dropping by an order of magnitude after the event, and an $\mathrm{O}^{8+} / \mathrm{O}^{7+}$ ratio which was low when $\mathrm{O}^{7+} / \mathrm{O}^{6+}$ was high but increased by a factor of twenty when $\mathrm{O}^{7+} / \mathrm{O}^{6+}$ fell. For the second possibility, described in panels $2 \mathrm{a}$ and $2 \mathrm{~b}$, we adopt the measured $\mathrm{O}^{7+} / \mathrm{O}^{6+}$ ratio and assume $\mathrm{O}^{8+} / \mathrm{O}^{7+}=0.57$ during the event as implied by the $\mathrm{O}^{8+}$ and $\mathrm{O}^{7+}$ lines ratio calculation in SCK04. This ratio is quite 

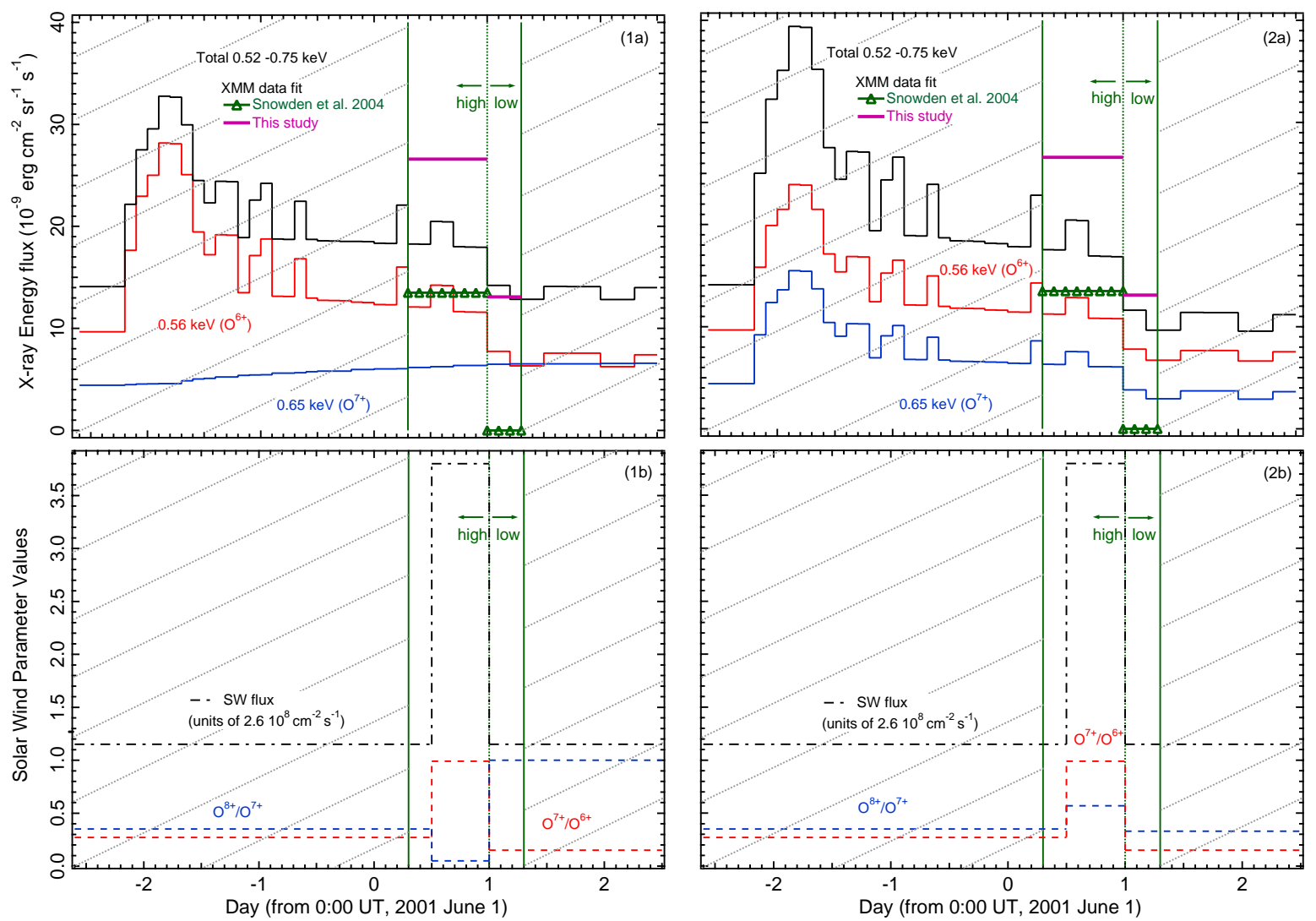

Fig. 8. Various parameters' temporal profiles, before, during and after the June 1, 2001 HDFN observation. Periods where no X-ray observations of HDFN are available are crosshatched in the graphs. Lower panels show SW input parameters: The dashed black line shows the proton flux enhancement, estimated as a step function, in units of SPFU (see text for further explanation). The dashed red line and the dashed blue line show the $\mathrm{O}^{7+} / \mathrm{O}^{6+}$ and the $\mathrm{O}^{8+} / \mathrm{O}^{7+}$ ratio evolution respectively. Left panel: $\mathrm{O}^{7+} / \mathrm{O}^{6+}$ and $\mathrm{O}^{8+} / \mathrm{O}^{7+}$ ratios derived from ACE measurements. Right panel: $\mathrm{O}^{8+} / \mathrm{O}^{7+}$ ratio implied by Snowden et al. analysis. Upper panels show X-ray emission temporal profiles for the two cases considered: The plain black line shows the simulation result for X-ray energy flux in the energy range $0.52-0.75 \mathrm{keV}$. The plain red and blue lines show X-ray energy fluxes for the two major spectral lines in this energy range: the O VII $0.56 \mathrm{keV}$ and O VIII $0.65 \mathrm{keV}$ lines respectively. The green horizontal lines show average high and low heliospheric energy fluxes in the energy range $0.52-0.75 \mathrm{keV}$ by XMM data fit, assuming there is no heliospheric contribution in the low X-ray emission state (SCK04). The purple horizontal lines show equivalent averages assuming that there is no cosmic background contribution, only particle background (this study). Energy fluxes are all presented in units $10^{-9} \mathrm{ergs} \mathrm{cm}^{-2} \mathrm{sr}^{-1} \mathrm{~s}^{-1}$.

superior to the normal value $(0.35)$ but not unreasonably so and moreover it involves a similar $\mathrm{O}^{7+}$ and $\mathrm{O}^{8+}$ evolution throughout the event. After the event cut-off we consider a $\mathrm{O}^{8+} / \mathrm{O}^{7+}$ ratio of 0.33 which implies again a similar decrease of $\mathrm{O}^{7+}$ and $\mathrm{O}^{8+}$ which is the most frequent case in solar events.

The red and blue curves show the X-ray lightcurves for the $\mathrm{O}$ VII line at $0.56 \mathrm{keV}$ and the O VIII line at $0.65 \mathrm{keV}$ respectively. The plain black curve shows the simulated lightcurve for total $\mathrm{X}$-ray emission in the $0.52-0.75 \mathrm{keV}$ energy range.

Figure 7, demonstrates that the LOS is affected by the SW enhancement about $3.0 \mathrm{~d}$ before the begining of observations, which corresponds to around day -2.6 on our lightcurve. The Xray emissions contained in the two lines evolve in very similar ways in the two models. Calculated with ACE data (left panel), it rises suddenly by about $130 \%$ half a day later, to reach a maximum on day -1.8 from June 1, 0:00 UT. Then, a first drop occurs on day -1.0 at $\sim 57 \%$ of the maximum value and a second at $\sim 40 \%$ of the maximum value on day +1 . This is exactly the moment the SW enhancement ends. The emission calculated with the $\mathrm{O}^{8+} / \mathrm{O}^{7+}$ ratio (right panel) implied by the spectral lines rises and falls at exactly the same intervals and more steeply. The emission rises by $\sim 150 \%$, then decreases by $53 \%$ and at the last cut-off, on day +1 , is stabilized at $30 \%$ of the maximum value.
The main difference between the two possible scenarios lies in the evolution of the two OVII and OVIII lines. With the second set of conditions, the lines behave in similar ways, decreasing in intensity by factors 1.6 and 1.94 respectively. The mean high intensities are $11.5 \times 10^{-9} \mathrm{erg} \mathrm{cm}^{-2} \mathrm{sr}^{-1} \mathrm{~s}^{-1}$ for O VII and $6.5 \times 10^{-9} \mathrm{erg} \mathrm{cm} \mathrm{cm}^{-2} \mathrm{sr}^{-1} \mathrm{~s}^{-1}$ for O VIII, and the mean low $7.26 \times 10^{-9}$ erg $\mathrm{cm}^{-2} \mathrm{sr}^{-1} \mathrm{~s}^{-1}$ for $\mathrm{O}$ VII and $3.38 \times 10^{-9} \mathrm{erg} \mathrm{cm}^{-2} \mathrm{sr}^{-1} \mathrm{~s}^{-1}$ for O VIII. According to the first scenario the OVIII line is nearly constant varying between $6.28 \times$ $10^{-9} \mathrm{erg} \mathrm{cm}^{-2} \mathrm{sr}^{-1} \mathrm{~s}^{-1}$ and $6.5 \times 10^{-9} \mathrm{erg} \mathrm{cm}^{-2} \mathrm{sr}^{-1} \mathrm{~s}^{-1}$ ), and the OVII line decreases strongly from $12.5 \times 10^{-9} \mathrm{erg} \mathrm{cm}^{-2} \mathrm{sr}^{-1} \mathrm{~s}^{-1}$ to $7.04 \times 10^{-9} \mathrm{erg} \mathrm{cm}^{-2} \mathrm{sr}^{-1} \mathrm{~s}^{-1}$.

Because there are no observations of the HDFN before day 0.3 after June 1, 0:00 UT, our comparison with the data is limited to the interval $0.3: 1.3$ day from June 1 , where the second cutoff occurs. The simulated lightcurves at the beginning of XMM observation are already at a high mean level, and present faint variations until the moment the SW enhancement cuts-off, on day +1 . This is exactly the temporal pattern of the $0.52-0.75 \mathrm{keV}$ emission recorded by XMM, as can be seen in Fig. 1 of Snowden et al. This emission drops by a factor of about 1.5.

To proceed further requires additional assumptions on the heliospheric and cosmic background radiations for this direction. We consider two extreme cases. 
In the first case we assume, following Snowden et al. that the high emission level is significantly contaminated by the heliosphere while during the low level period this local emission is negligible and most of the signal is of cosmic origin (except for the particle background). The difference between the two high and low spectra provides a measurement of the heliospheric contribution during the event. Since the heliospheric contribution is contained in lines only, and is made of the OVII + OVIII lines in the $0.52-0.75 \mathrm{keV}$ range, it amounts to $13.5 \times 10^{-9}$ ergs $\mathrm{cm}^{-2} \mathrm{sr}^{-1} \mathrm{~s}^{-1}$ as derived from line fitting by SCK04 (Tab. 2, SCK04, sum of the two O lines). This absolute level is significantly smaller than the model estimates of about $18.8 \times 10^{-9} \mathrm{erg} \mathrm{cm}^{-2} \mathrm{sr}^{-1} \mathrm{~s}^{-1}$ and $18.0 \times 10^{-9} \mathrm{erg} \mathrm{cm}^{-2} \mathrm{sr}^{-1} \mathrm{~s}^{-1}$ for the first and second case respectively. In contrast, the low level is effectively zero. We have plotted in Fig. 8 the two values (13.5 and $0 \times 10^{-9} \mathrm{erg} \mathrm{cm}^{-2} \mathrm{sr}^{-1} \mathrm{~s}^{-1}$ ). Despite the fluctuations on the measured intensities they are inconsistent with the simulations, which imply a relative decrease by less than a factor of 2 (more precisely 1.4 and 1.7 respectively).

If we assume that there is no signal other than from the heliosphere (except for the particle background) we can use the background subtracted high and low XMM spectra, derived from Fig. 3 of SCK04, to estimate the heliospheric contributions. We obtain high to low ratios of 1.66 for the OVII line and 2.28 for the OVIII line. These ratios match those obtained in the second scenario but the total high intensity is $25 \times 10^{-9} \mathrm{erg} \mathrm{cm}^{-2} \mathrm{sr}^{-1} \mathrm{~s}^{-1}$, much higher than the model predictions. However in view of the many simplifying assumptions, we cannot conclusively exclude a model with a very low cosmic background.

Our conclusion here is that very likely the actual situation is somewhere between the two extreme cases. Still, the good agreement on the light curves and the overall intensity for model and data in the second case strongly suggests that (i) heliospheric $\mathrm{X}$-rays are the main contributor to the observed enhancement and (ii) even the LOW spectrum contains a large fraction of heliospheric emission.

\section{Discussion}

We have performed a parametric study of the heliospheric X-ray background emission and an analysis of the multiple factors which can influence the emission level. We have demonstrated the complex response to these factors, which include the date of observation (through the difference between the earth longitude and the interstellar wind axis longitude), the solar cycle phase (through the $\mathrm{H}$ and $\mathrm{He}$ densities which vary significantly with the phase), the solar wind type (through the high ion absolute and relative abundances), and finally the solar wind history (through the potentially very strong solar wind enhancements and abundance variations). The contribution to the signal generated in the inner heliosphere by secondary collisions has been shown to be negligible. The contribution to the signal of the external heliosphere (heliosheath and tail) is estimated and found to be small, except in the tail direction for some particular ions. This type of model results should allow an estimate of the expected range for the emission for a given direction of sight and date.

We have modelled in a very simplified way the temporal variation of the signal in the case of a sudden and temporary solar wind increase, and made a data/model comparison in the case of the XMM-HDFN. It is possible to reproduce the intensity level and the temporal evolution of the line intensities, although no solution has been found here matching perfectly all the data simultaneously. It is likely however from this study that a large fraction of the signal is of heliospheric origin, including in the post-event (LOW) period of time. This is in agreement with the results of Cravens et al. (2001), based on correlations with the solar wind, and of Lallement (2004) based on the Local Bubble emission pattern.

It is clear from Fig. 7 that each specific geometry will produce a different temporal variation. A LOS at 180 degrees from the HDFN direction would have had a totally different light curve, for the same solar wind history. Such complex temporal variations preclude a good correlation on small time scales between the X-ray emission and the locally measured solar wind. On the other hand, this diversity can be useful because the combination of numerous examples such as the above one may allow constraints on the actual local and cosmic contributions.

To reach this goal a number of improvements in the modelling have to be worked out. We have been using here a single fluid model for the IS $\mathrm{H}$ density, but we know that $\mathrm{H}$ atoms are separated in the outer heliosphere in two populations with different temperature, velocity and arrival direction (e.g. Lallement et al. 2005). Two population models should be developed. More important, in our simulation the eruptive region is stationary in the Sun's frame, like a garden hose, and apart from one enhanced stream we have only used simplified stationary states of solar cycle minimum and maximum conditions. Realistic models should include 3D solar wind hourly data (ideally from different vantage points) as well as precise measurements of the high ion relative abundances. The exact 3D observation geometry should be, as well, taken into account in the model, which requires the knowledge of the active region geometry. In parallel, a more precise time-dependent model of the neutral distributions, consistent with the solar wind flux and velocity variations is needed.

Acknowledgements. We wish to thank John Raymond for useful discussions and valuable suggestions which led to the correction of a mistake. R.L. and D.K., V.I. acknowledge funding by CNRS and RFBR respectively under PICS contract 3205. A.D. and V.K. acknowledge NASA for support through grant NNG04GD57G.

\section{References}

Auchère, F., Cook, J. W., Newmark, J. S., et al. 2005, ApJ, 625, 1036 Baranov, V. B., \& Malama, Yu. G. 1993, JGR, 98, 15157

Beiersdorfer, P., Lisse, C. M., Olson, R. E., Brown, G. V., \& Chen, H. 2001, ApJ, 549, L147

Beiersdorfer, P., Boyce, K. R., Brown, G. V., et al. 2003, Science, 300, 1558 Beijers, J. P. M., Hoekstra, R., \& Morgenstern, R. 1994, Phys. Rev. A, 49, 363 Bliman, S., Barany, A., Bonnefoy, M., et al. 1992, J. Phys. B, 25, 2065 Bonnet, J. J., Fleury, A., Bonnefoy, M., et al. 1985, J. Phys. B, 18, L23 Bowyer, S., \& Vikhlinin, A. 2004, JKAS, 37, 579

Costa, J., Lallement, R., Quémerais, E., et al. 1999, A\&A, 349, 660

Cox, D. P. 1998, Lect. Notes Phys., 506, 121

Cravens, T. E. 1997, GeoRL, 24, 105

Cravens, T. E. 2000, ApJ, 532, L153

Cravens, T. E., Robertson, I.P., \& Snowden, S. L. 2001, JGR, 106, 24883

Dijkkamp, D., Gordeev, Yu S., Brazuk, A., Drentje, A. G., \& Heer, F. J. 1985a, J. Phys. B, 18, 737

Dijkkamp, D., Ciric, D., de Boer, A., de Heer, F. J., \& Vlieg, E. 1985b, J. Phys. B, 18,4763

Freyberg, M. J. 1994, Ph.D. Thesis, Technische Univ. München

Freyberg, M. J. 1998, AN, 319, 93

Fritsch, W., \& Lin, C. D. 1996, J. Phys. A, 54, 4931

Gloeckler, G., Mbius, E., Geiss, J., et al. 2004, A\&A, 426, 845

Greenwood, J., Williams, I. D., Smith, S. J., \& Chutjian, A. 2001, Phys. Rev. A, 63, 062707

Gruntman, M., Fahr, \& H. J. 1998, GeoRL, 25, 1261

Häberli, R. M., Gombosi, T. I., DeZeeuw, D. L., Combi, M. R., \& Powell, K. G. 1997, Science, 276, 939

Harel, C., \& Jouin, H. 1992, J. Phys. B, 25, 221

Harel, C., Jouin, H., \& Pons, B. 1998, Atomic Data and Nuclear Data Tables, 68, 279

Ishii, K., Itoh, A., \& Okuno, K, 2004, Phys. Rev. A, 70, 042716 
Iwai, T, Kaneko, Y, Kimura, M., et al. 1982, Phys. Rev. A, 26, 105 Izmodenov, V., \& Alexashov, D. 2003, Astron. Lett., 29, 58. Translated from Pis'ma v Astronomicheskii Zhurnal, 2003, 29, 69

Izmodenov, V., Malama, Y., Gloeckler, G., \& Geiss, J. 2004, A\& A, 414, L29

Kharchenko, V., \& Dalgarno, A. 2000, JGR, 105, 18351

Kharchenko, V., \& Dalgarno, A. 2001, ApJ, 554, L99

Kharchenko, V., Rigazio, M., Dalgarno, A., \& Krasnopolsky, V. 2003, ApJ, 585, L73

Krasnopolsky, Vladimir, 1997, Icarus, 128, 368

Kuntz, K. D., \& Snowden, S. L. 2000, ApJ, 543, 195

Lallement, R. 1999, Solar Wind 9 Am. Inst. Phys. Conf. Proc. 471, 205

Lallement, R. 2004, A\&A, 418, 143

Lallement R., \& Bertaux J. L. 1990, A\&A, 231, L3

Lallement, R., Bertaux, J. L., Dalaudier, F. 1985a, A\&A, 150, 21

Lallement, R., Bertaux, J. L., \& Kurt, V. G. 1985b, JGR, 90, 1413L

Lallement, R., Raymond, J. C., Vallerga, et al. 2004, A\&A, 426, 875

Lallement, R., Quémerais, E., Bertaux, J. L., et al. 2005, Science, 307, 1447

Lee, T.-G., Hesse, M., Le, A.-T., \& Lin, C. D. 2004, Phys. Rev. A, 70, 012702

Lisse, C. M., Dennerl, K., Englhauser, et al. 1996, Science, 274, 205

Liu, C.-N., Cheng, S.-C., Le, A.-T., \& Lin, C. D. 2005, Phys. Rev. A, 72, 012717

Malama, Yu. G., Izmodenov, V. V., \& S. V. Chalov, 2006, A\& A, 445, 693

McMullin, D. R., Bzowski, M., Möbius, E., et al. 2004, A\&A, 426, 885

Pepino, R., Kharchenko, V., Dalgarno, A., \& Lallement, R. 2004, ApJ, 617, 1347

Phaneuf, R. A., et al. 1987, ORNL-6090
Quémerais, E., Lallement R., Ferron S., et al. 2006, JGR, in press

Richter, K., \& Solov'ev 1993, Phys. Rev. A, 48, 432

Rigazio, M., Kharchenko, V., \& Dalgarno, A. 2002, Phys. Rev. A, 66, 64701

Robertson, I. P., \& Cravens, T. E. 2003a, GeoRL, 30, 8, 22,a

Robertson, I. P., \& Cravens, T. E. 2003b, JGRA, 108, LIS6,b

Robertson, I. P., Cravens, T. E., Snowden, S., \& Linde, T. 2001, Sp. Sci. Rev., 97,401

Schwadron, N. A., \& Cravens, T. 2000, ApJ, 544, 558

Shimakura, N., Koizumi, S., Suzuki, S., \& Kimura, M. 1992, Phys. Rev. A, v45, 7876

Smith, E. J., Marsden, R. G., Balogh, A., et al. 2003, Science, 302, 1165

Snowden, S. L., Freyberg, M. J., Kuntz, K. D., Sanders, W. T. 2000, ApJS, 128, 171

Snowden, S. L., Collier, M. RM, \& Kuntz, K. D. 2004, ApJ, 610, 1182

Suraud, M. G., Hoekstra, R., de Heer, F. J., Bonnet, J. J., \& Morgenstern, R. 1991, J. Phys. B, 24, 2543

Von Steiger, R., Schwadron, N. A., Fisk, L. A., et al. 2000, JGR, 105, A12, 27217

Vallerga, J., Lallement, R., Lemoine, M., Dalaudier, F., \& McMullin, D. 2004, A\&A, 426, 855

Wargelin, B. J., Markevitch, M., Juda, et al. 2004, ApJ, 607, 1, 596

Witte M. 2004, A\&A, 426, 835

Woods, T. E., Kent Tobiska, W., Rottman, G. J., \& Worden, J. R. 2000, JGR, 105,27195

Wu, W., Giese, J. P., Chen, Z., et al. 1994, Phys. Rev. A, v50, 502 


\section{Online Material}




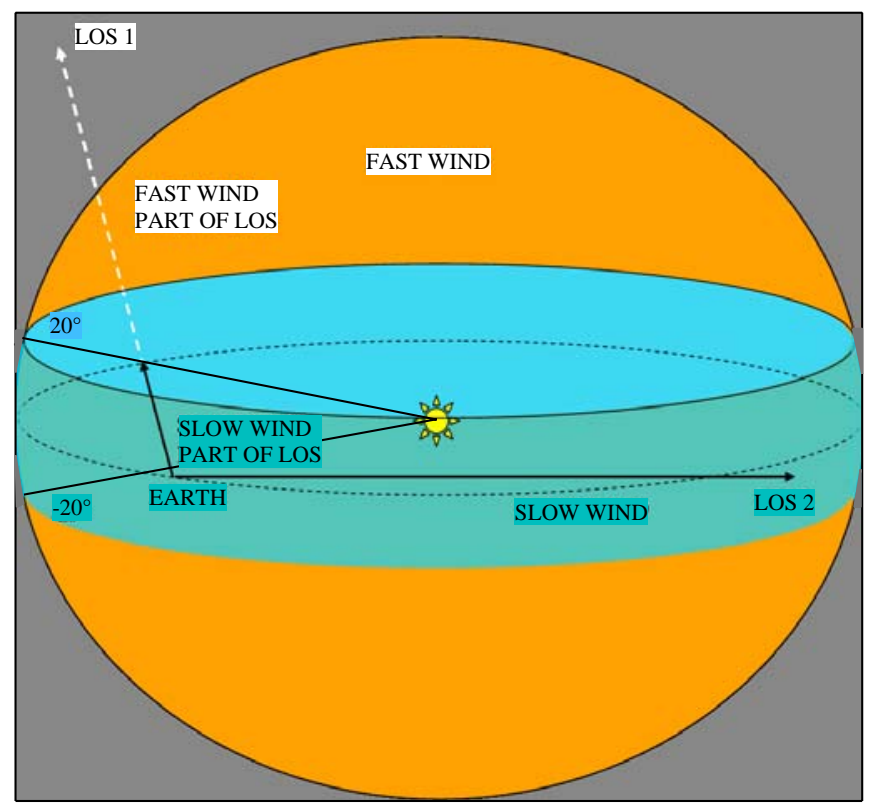

Fig. 9. Line of Sight (LOS) Geometry.
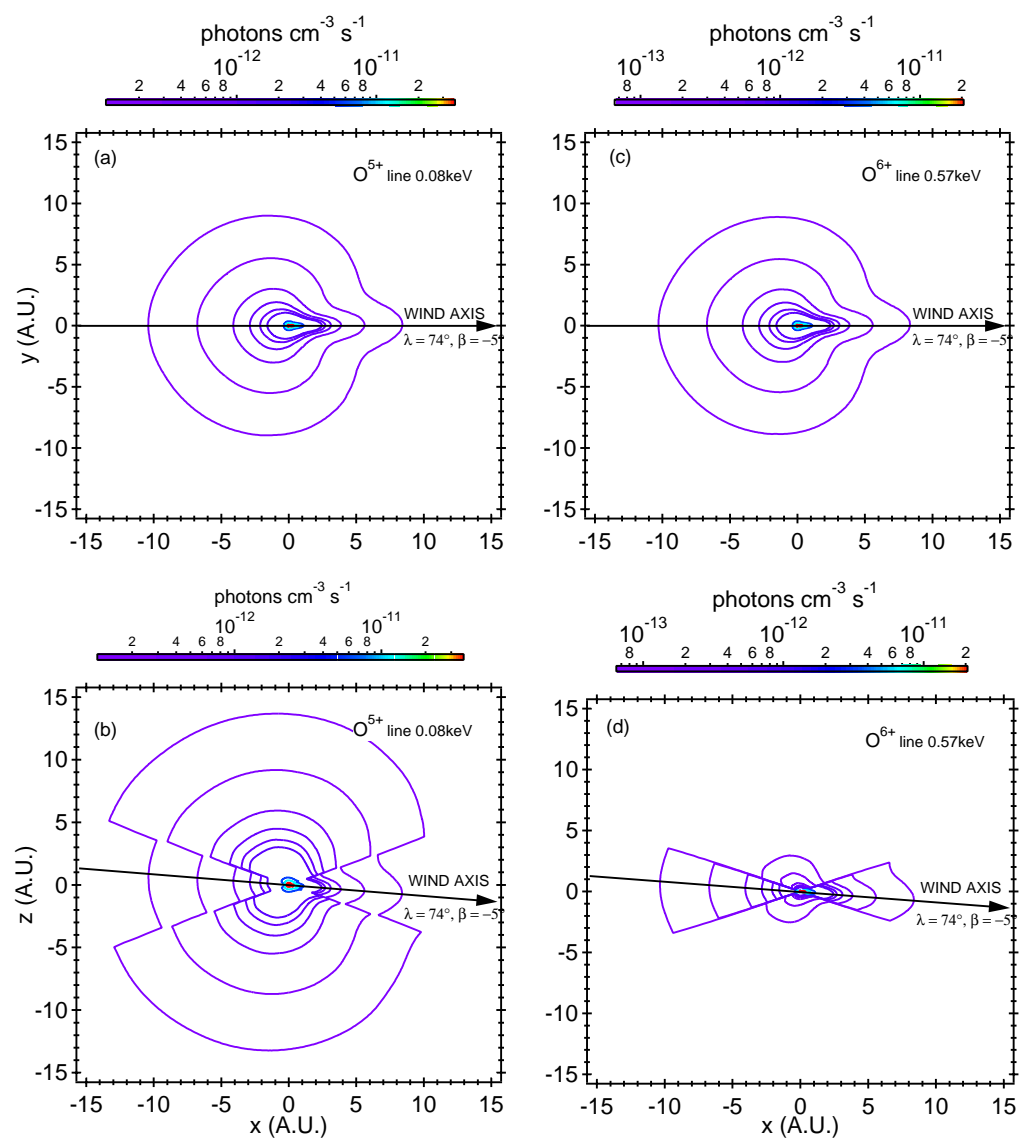

Fig. 10. Iso-emissivity contour maps up to $15 \mathrm{AU}$ for solar minimum conditions. Left column corresponds to the sum of the ion $\mathrm{O}^{5+}$ lines at 0.072 and $0.082 \mathrm{keV}$ and right column to the sum of the ion $\mathrm{O}^{6+}$ lines at $0.561,0.569$ and $0.574 \mathrm{keV}$. Top panels correspond to the plane $(x, y)$ defined by the vectors $\left(74^{\circ},-5^{\circ}\right)$ and $\left(-16^{\circ}, 0^{\circ}\right)$. Bottom panels correspond to the plane $(x, z)$ containing the IS He wind axis $\left(74^{\circ},-5^{\circ}\right)$ and the ecliptic axis. The color scale is in units of photons $\mathrm{cm}^{-3} \mathrm{~s}^{-1}$. 
Koutroumpa et al.: Heliospheric X-ray emission, Online Material p 3

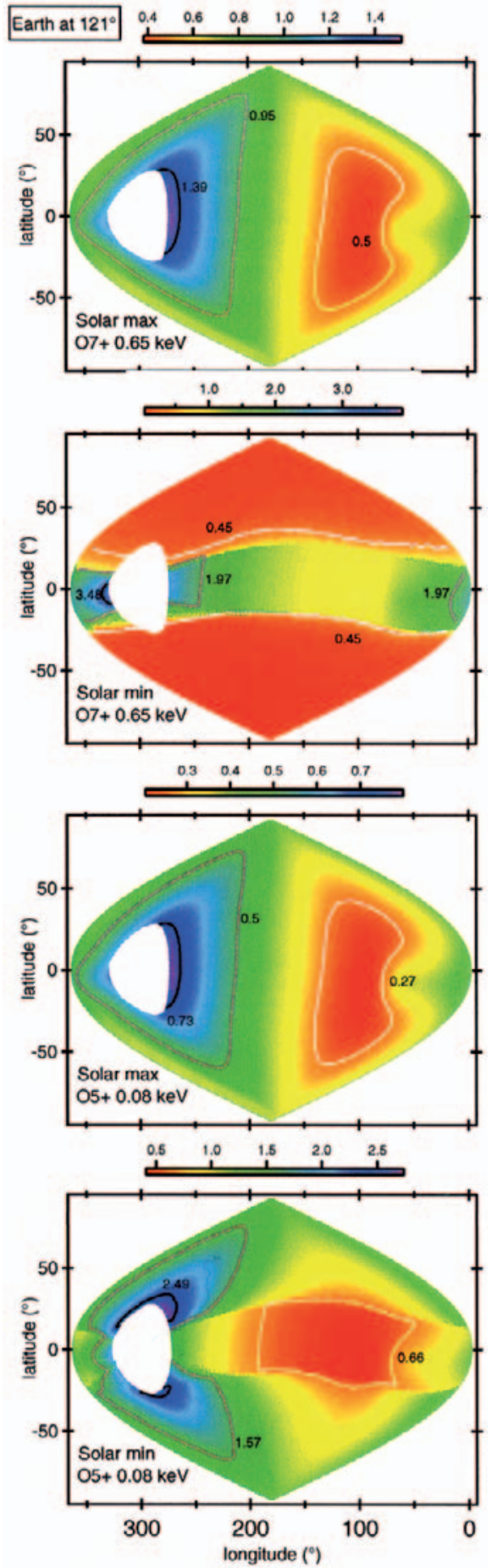

Fig. 11. Solar maximum and solar minimum full sky monochromatic emission maps. The two lines here are the $0.65 \mathrm{keV}\left(\mathrm{O}^{7+}\right)$ and the $0.08 \mathrm{keV}\left(\mathrm{O}^{5+}\right)$. The observer is situated at $121^{\circ}$ ecliptic longitude. The color scale is in units of $10^{-9} \mathrm{erg} \mathrm{cm}^{-2} \mathrm{sr}^{-1} \mathrm{~s}^{-1}$, red colour corresponding to minimum and blue to maximum values. The masked area corresponds to the $20^{\circ} \times 20^{\circ}$ region around the solar disk. The map is shown in ecliptic coordinates.

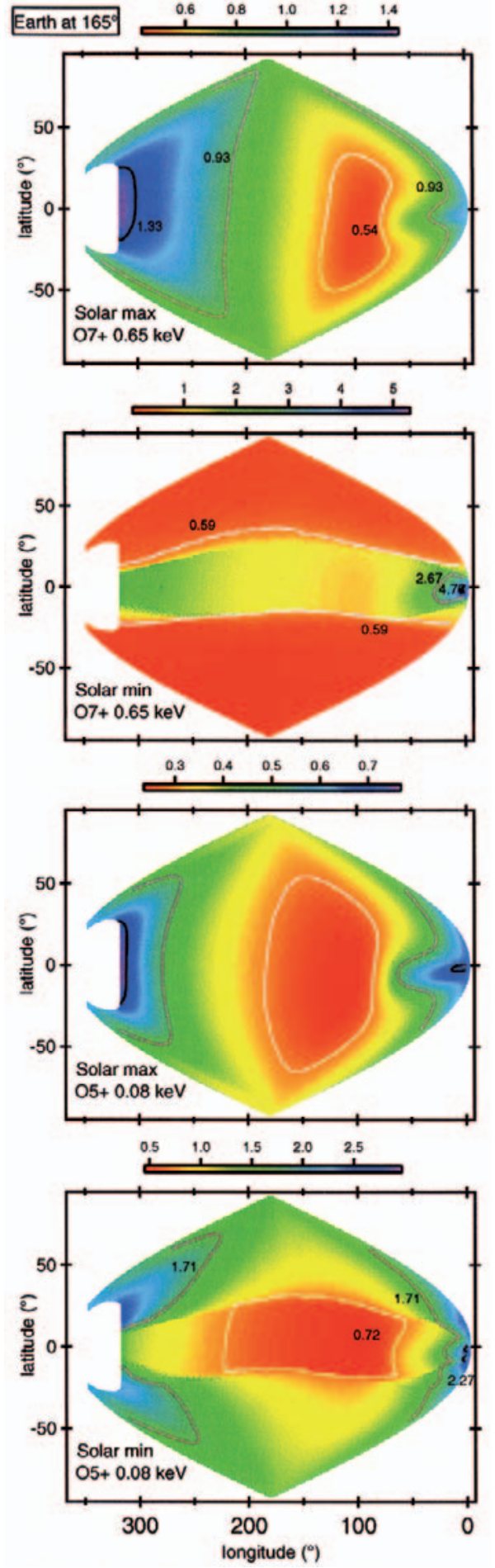

Fig. 12. Same as Fig. 11 for an observer at $165^{\circ}$ ecliptic longitude. The color scale is in units of $10^{-9} \mathrm{erg} \mathrm{cm}^{-2} \mathrm{sr}^{-1} \mathrm{~s}^{-1}$, red colour corresponding to minimum and blue to maximum values. 
Koutroumpa et al.: Heliospheric X-ray emission, Online Material $p 4$

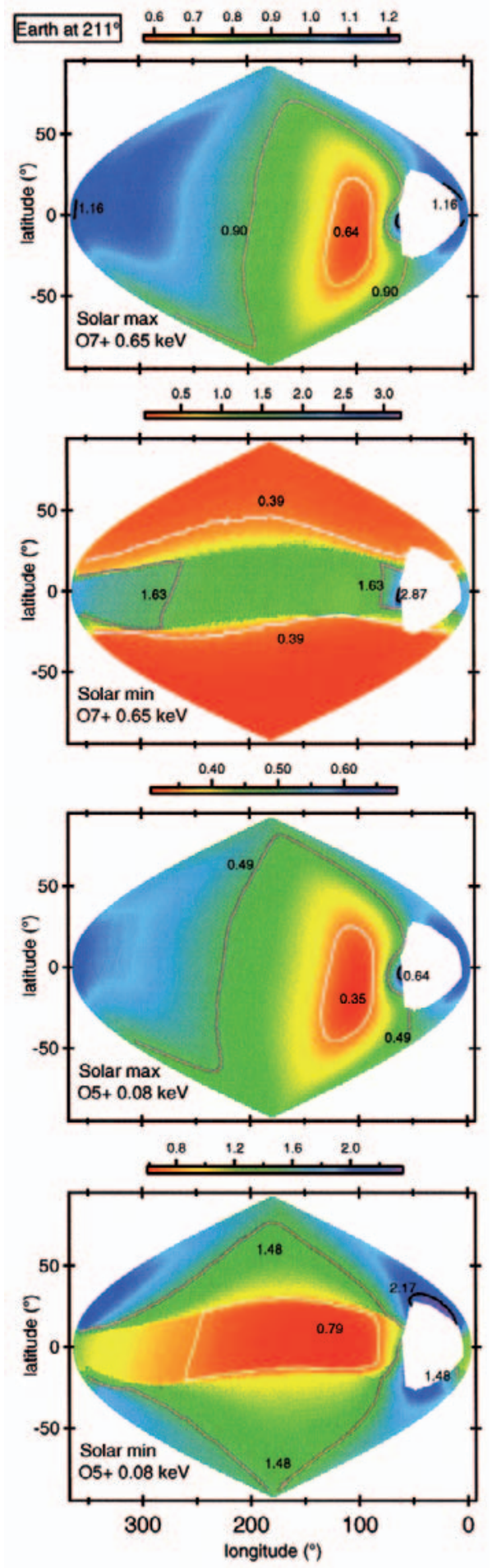

Fig. 13. Same as Fig. 11 for an observer at $211^{\circ}$ ecliptic longitude. The color scale is in units of $10^{-9} \mathrm{erg} \mathrm{cm}^{-2} \mathrm{sr}^{-1} \mathrm{~s}^{-1}$, red colour corresponding to minimum and blue to maximum values. 